\title{
Intracranial Metastatic Disease: Present Challenges, Future Opportunities
}

\begin{abstract}
Alyssa Y. $\mathrm{Li}^{1 \dagger}$, Karolina Gaebe ${ }^{1 \dagger}$, Katarzyna J. Jerzak ${ }^{1,2}$, Parneet K. Cheema ${ }^{3}$, Arjun Sahgal ${ }^{1,4}$ and Sunit Das ${ }^{1,5^{*}}$

${ }^{1}$ Institute of Medical Science, Faculty of Medicine, University of Toronto, Toronto, ON, Canada, ${ }^{2}$ Division of Oncology, Department of Medicine, Sunnybrook Health Sciences Centre, University of Toronto, Toronto, ON, Canada, ${ }^{3}$ Division of Oncology, William Osler Health System, Brampton, ON, Canada, ${ }^{4}$ Department of Radiation Oncology, Sunnybrook Health Sciences Centre, University of Toronto, Toronto, ON, Canada, 5 Division of Neurosurgery, St. Michael's Hospital, University of Toronto, Toronto, ON, Canada
\end{abstract}

Intracranial metastatic disease (IMD) is a prevalent complication of cancer that significantly limits patient survival and quality of life. Over the past half-century, our understanding of the epidemiology and pathogenesis of IMD has improved and enabled the development of surveillance and treatment algorithms based on prognostic factors and tumor biomolecular characteristics. In addition to advances in surgical resection and radiation therapy, the treatment of IMD has evolved to include monoclonal antibodies and small molecule antagonists of tumor-promoting proteins or endogenous immune checkpoint inhibitors. Moreover, improvements in the sensitivity and specificity of imaging as well as the development of new serological assays to detect brain metastases promise to revolutionize IMD diagnosis. In this review, we will explore current treatment principles in patients with IMD, including the emerging role of targeted and immunotherapy in select primary cancers, and discuss potential areas for further investigation.

Keywords: intracranial metastatic disease (IMD), brain metastases, targeted therapy, immunotherapy, neurosurgery, minimally invasive surgery, radiation therapy, screening

\section{INTRODUCTION}

The development of intracranial metastatic disease (IMD) is a frequent and serious complication of cancer, affecting nearly $30 \%$ of cancer patients (1). The incidence of IMD is expected to increase as our population ages and as advancements in primary cancer therapy result in longer disease survival (1). IMD has been observed to disproportionately affect patients with certain primary cancers, including lung cancer (19.6\%), melanoma (6.4\%), renal cell carcinoma (4.2\%), breast cancer (3.1\%), and colorectal cancer (1.4\%), reflecting possible organotropy in IMD or successes with local disease control (2). Indeed, among patients with brain metastases ( $\mathrm{BrM})$, lung cancer, breast cancer, and melanoma account for nearly $60 \%, 11 \%$, and $6 \%$ of all primary cancers in some studies (2).

The impact of IMD on morbidity and mortality of patients with cancer is significant. The median time to diagnosis of IMD has been reported to be 5.2 months following primary cancer diagnosis, suggesting that a large proportion of patients may already have $\mathrm{BrM}$ at the time their primary cancer is diagnosed (2). Given the high prevalence of IMD and the short median survival of 3.7 months 
following IMD diagnosis, there is interest in developing screening strategies and tools to identify patients with IMD (2). Magnetic resonance imaging (MRI) remains the most commonly used modality for the diagnosis of $\mathrm{BrM}$ and leptomeningeal disease (3). Historically, MRI evaluation has been prompted by the development of neurological symptoms, including headache, seizure, and altered mental status, in patients with cancer. Several algorithms have since been created to identify asymptomatic, high-risk patients who would benefit from MRI screening at the time of their primary cancer diagnosis (3). Imaging and serum biomarkers of IMD to allow for earlier and more cost-effective diagnosis of IMD are under investigation, however, their role in IMD surveillance remains to be fully elucidated $(4,5)$.

At present, the most common local treatments for IMD are surgical resection, whole brain radiation therapy (WBRT), and stereotactic radiosurgery (SRS). The Graded Prognostic Assessment (GPA) is often used to estimate survival for patients with BrM and is based on prognostic factors including Karnofsky performance score (KPS), age, presence of extracranial metastases, number of BrM, and tumor subtype, depending on the primary tumor histology (6). In some treatment algorithms, patients with greater IMD burden or poorer performance status are directed toward WBRT, while younger patients with fewer intracranial metastases may be directed toward treatment with surgical resection or SRS (7). Advances in surgical technique and delivery of radiation have resulted in improved survival estimates for patients with IMD from less than six months over fifteen years ago to 8-16 months today $(6,8-11)$.

Recent data have supported a role for systemic targeted therapies and immunotherapies in the treatment of select patients with IMD, including but not limited to those with non-small cell lung cancer (NSCLC), breast cancer, and malignant melanoma $(12,13)$. There remains, however, a clear clinical need for further investigation to develop more effective screening and treatment for IMD. In this review, we outline and describe recent and current efforts to improve outcomes in patients with IMD of the brain through novel therapeutics, improved surveillance, and prevention.

\section{EMERGING TREATMENTS FOR IMD}

Traditionally, prognosis for patients with IMD has been extremely poor. However, modern treatments have significantly improved survival and quality of life (QoL) in these patients. The main goals of IMD therapy include lengthening survival, diminishing or controlling IMD burden, minimizing adverse events associated with IMD development and treatment, as well as improving cognition and QoL.

\subsection{Surgical Resection and Minimally Invasive Surgery}

Surgery has been a long-standing modality in the treatment of BrM. In contrast to other forms of IMD management, surgical resection can offer immediate relief of mass effect and neurological symptoms caused by compression and edema, while providing tissue for diagnostic purposes $(14,15)$. Risks associated with neurosurgery include iatrogenic neurological injury, hemorrhage, infection, and seizure (16, 17). Recent literature has also confirmed a long-standing concern that surgery holds a risk of leptomeningeal disease recurrence, likely related to unintentional local cell seeding during the process of surgical resection (18). However, with modern techniques, surgical resection is typically safe and recovery from surgery often short, making surgery an appealing option, particularly for patients with a large brain lesion, limited number of BrM, and well controlled systemic disease (19). In the 1990s, two landmark randomized controlled trials (RCTs) demonstrated that surgical resection compared with needle biopsy or no surgery prior to WBRT decreased local recurrence, improved median survival, and resulted in more rapid and sustained functional independence (20, 21). In contrast, a similar study by Mintz et al., which included many patients with progressive extracranial disease and lower performance status, found no survival benefit with the addition of surgical resection to WBRT (22). A recent systematic review and meta-analysis found that the literature supports surgical resection followed by postoperative WBRT in patients with good performance status (KPS $\geq 70$ ), controlled systemic disease, and a single brain lesion (23).

While surgical resection of a single BrM in patients with well controlled systemic disease is well-established in clinical practice, the role of surgery in the treatment of patients with limited BrM (2-4 lesions) or deep BrM remains a topic of debate $(22,24)$. In current clinical practice, these patients are more often treated with SRS. A few retrospective studies have reported equivalence of outcomes between groups treated with surgery and SRS for limited BrM (25-27). SRS is less effective for larger lesions and is associated with delayed treatment effect, differences that have become more significant as improvements in systemic therapies have led to prolonged overall survival (OS) and highlighted the importance of achieving adequate IMD control to maintain QoL $(24,28)$. These factors, as well as the significant benefits observed with surgical resection in the management of single brain lesions, have prompted investigation into the role of surgery in patients with limited BrM or deep, large lesions (29-31).

Advances in surgical technique and intraoperative technologies may expand indications for surgical resection. Improvements in microsurgery, for example, through improved microscopic and endoscopic visualization, the use of "keyhole" techniques, and evolution away from the use of fixed retractors during surgery, have greatly reduced surgical morbidity and mortality, with recent studies reporting a complication rate between $2-9 \%(32-36)$. Further, the development of minimally invasive parafascicular surgery (MIPS), based on the use of a minimal-access tubular retractor advanced into the brain using stereotactic guidance, has allowed surgeons access to deep-seated lesions, while minimizing the iatrogenic injury that has been historically the cost of reaching these areas (37). Even in the setting of high-risk brain lesions, 
minimal complications were reported by Gassie et al. with the use of MIPS in 15 patients with deep-seated brain tumors: only one patient had decreased postoperative KPS and no patients developed local complications, such as stroke, infection, hemorrhage, or seizure (38). These results imply that surgical management of deep-seated, high-risk brain lesions may be feasible, however, clinical efficacy in comparison with other treatment approaches remains to be studied.

\subsection{Radiation Therapy}

WBRT has been a mainstay for the treatment of IMD since the middle of the $20^{\text {th }}$ century (39-41). Gains in patient survival with improvements in systemic therapies, however, have brought mounting concerns regarding the late toxicities of WBRT, particularly on neurocognitive function (42). Several modifications to the delivery of WBRT have since been investigated, for example, techniques that spare exposure to the hippocampus, or concurrent delivery of a neuroprotective agent (43-46). In current practice, WBRT remains the preferred treatment option for patients with multiple BrM, widely disseminated metastatic disease, or short life expectancy, and is used as prophylactic therapy for patients with small cell lung cancer (SCLC) in the absence of IMD (47).

\subsubsection{The Evolution of SRS in the Treatment of IMD}

A major improvement in the treatment of BrM has been SRS, which allows for focal delivery of high doses of radiation. For patients with limited (1-4) BrM, SRS has been shown to be an effective treatment to achieve local control of lesions within the cerebrum, cerebellum, and brainstem (48, 49). A secondary analysis of an RCT designed to investigate the addition of WBRT to SRS or surgical resection showed similar rates of local control following surgical resection and WBRT, compared with WBRT followed by cavity radiation with SRS (50). At present, we do not have RCT data that directly compare surgical resection and SRS in the setting of a single or limited BrM. Multiple studies, however, have investigated 1) the utility of adding SRS to WBRT; and 2) treatment with SRS as an alternative to WBRT.

Two seminal RCTs by Andrews et al. and Kodziolka et al. investigated the benefit of SRS boost following WBRT for patients with limited or multiple BrM. Both trials found that SRS improved OS (6.5 vs. 4.9 months, $\mathrm{p}=0.39$; and 11.0 vs. 7.5 months, $\mathrm{p}=0.03)$ as well as local control $(51,52)$. Given the neurotoxicity associated with WBRT, Aoyama et al. then asked if SRS was sufficient as a treatment for patients with limited BrM. One hundred thirty-two patients were randomized to SRS alone $(n=65)$ or WBRT and SRS $(n=67)$. Although higher rates of local tumor control were observed at 1 year in the group treated with WBRT and SRS compared with standalone SRS treatment ( $88.7 \%$ vs $72.5 \%, p=0.002)$, the addition of WBRT did not confer a statistically significant benefit on survival (median 7.5 vs. 8.0 months, $\mathrm{p}=0.42$ ) (53). A subsequent RCT by Chang et al. to evaluate neurocognitive decline in patients with limited BrM treated with SRS or WBRT plus SRS was halted prematurely when an interim analysis showed that patients randomized to receive WBRT plus SRS were at a significantly increased risk for neurocognitive decline at four months (54). Notably, their findings again showed a dissociation between achievement of local control and OS: both 1-year freedom from recurrence and risk of death were higher with combined WBRT and SRS (hazard ratio (HR) 2.47, $\mathrm{p}=0.0036 ; 27 \%$ vs. $73 \%, \mathrm{p}=0.0003$ ). The finding in this study that treatment with SRS alone conferred a greater survival benefit than bimodality treatment has received criticism as an artifact of higher burden of extracranial and intracranial disease in the WBRT plus SRS arm (55). Overall, these studies highlight the fact that many of these patients succumb to progression of their systemic disease.

A study by the European Organization for Research and Treatment of Cancer (EORTC) similarly found that WBRT improved local control at 2 years compared with observation only after SRS ( $31 \%$ vs. $19 \%, \mathrm{p}=0.040)$. In line with the findings by Aoyama and colleagues, however, no OS difference was observed between the SRS alone and WBRT plus SRS arms (SRS only: 10.7 months, WBRT plus SRS: 10.9 months, $\mathrm{p}=0.89$ ). Further, improved tumor control with the addition of WBRT did not lengthen the median duration of functional independence (SRS only: 10.0 months, WBRT plus SRS: 9.5 months, $\mathrm{p}=0.71$ ) (56). In fact, QoL was better in the SRS-only arm (57). In a separative analysis of this trial, Kim et al. found that, compared with upfront WBRT, SRS alone was more cost-effective in patients with one to three IMD lesions (58). Meta-analyses by Tsao et al. and Sahgal et al. concluded that, while WBRT improved local and distant brain control compared with SRS alone (HR 2.61, p <0.0001, and HR 2.15, p <0.00001, respectively), treatment with SRS alone is associated with longer survival in patients age $\leq 50$ years ( 13.6 vs. 8.2 months), and that there is no difference in survival with the addition of WBRT to SRS in patients age $>50$ years, compared to SRS alone (10.1 vs. 8.6 months, respectively) $(59,60)$. A subsequent trial in which patients with limited melanoma BrM treated with SRS were randomized to adjuvant WBRT showed no clinical benefit with the addition of WBRT to local therapy on distant intracranial control, survival, or preservation of performance status (61). Consequently, in a statement released by the American Society for Radiation Oncology as part of its Choosing Wisely campaign, addition of WBRT to SRS is no longer recommended for patients with limited number of BrM (62). Conversely, WBRT remains standard of care in patients with multiple ( $\geq 4 \mathrm{BrM}$ ). More recent data have supported a possible role for SRS in the treatment of patients with multiple $(\leq 10)$ BrM. A prospective observational study of nearly 1200 Japanese patients found similar rates of OS in patients treated with SRS for two to four lesions compared with SRS for five to ten lesions (HR 0.79, p=0.78) (63).

\subsubsection{Late Adverse Effects Following SRS}

Although SRS is generally well-tolerated, patients can experience adverse effects, most notably radiation necrosis, which negatively affects QoL and morbidity. Radiation necrosis develops in 5-25\% of patients following SRS treatment and constitutes a late adverse treatment effect that can occur months to years after SRS has been completed (64-70). Patients affected by radiation necrosis can present with a variety of symptoms ranging from asymptomatic to symptoms of increased intracranial pressure, 
including headaches, seizures, or cognitive/neurological decline (71). Diagnosis of radiation necrosis is challenging, as radiation necrosis and recurrent tumor lesions present with similar features on conventional imaging. Ultimately, biopsies of suspicious lesions are required to establish a definite diagnosis. While glial cell and vascular injury have been postulated as potential underlying etiologies for radiation necrosis, damage following SRS is directly related to radiation dose per fraction administered (72-74). Additional risk factors include prior radiation (in particular SRS) to the same site, larger lesion size, or receipt of targeted or immunotherapy $(64,75-77)$. In one study, SRS to the same lesion was associated with an increased risk of radiation necrosis at 1 year when compared with prior WBRT, concurrent WBRT, or no prior radiation $(20 \%, 4 \%, 8 \%$, and $3 \%$, respectively) (77). Blonigen et al. also demonstrated that the risk of radiation necrosis was higher when the volume of brain parenchyma receiving more than 10 Gy or 12 Gy exceeded $10.5 \mathrm{~cm}^{3}$ or $7.9 \mathrm{~cm}^{3}$, respectively (64). In another study of 480 patients secondary to NSCLC, melanoma, or renal cell carcinoma, the risk of radiation necrosis following SRS treatment was 2.5 times higher in patients who received prior immunotherapy, further illustrating the multi-faceted nature of radiation necrosis (78). Management of radiation necrosis can be conservative in the absence of symptoms, or can involve corticosteroids, bevacizumab, surgical resection, or laser interstitial therapy for symptom control (79-82). Radiation necrosis is a challenging complication of SRS treatment and should be considered as a potential complication following SRS.

\subsubsection{Approaches to Limit Cognitive Impacts of WBRT}

Given the neurotoxicity associated with WBRT, several strategies to limit the cognitive impacts of WBRT have been investigated. Our advancing understanding of the mechanisms underlying radiation-induced injury has led to the development of pharmacological agents that modulate the brain's sensitivity to radiation-induced effects. One such agent is memantine, a drug that is used in the treatment of Alzheimer's dementia and that helps prevent vascular injury. The efficacy of memantine in preventing radiation-induced cognitive dysfunction was investigated in a RCT (RTOG 0614) of 508 patients treated with WBRT and either memantine $(20 \mathrm{mg} /$ day $)$ or placebo, both initiated within three days of WBRT for 24 weeks. There were no differences in OS and progression-free survival (PFS) between the two arms, and memantine did not cause additional toxicity. Although no statistically significant differences in terms of the Hopkins Verbal Learning Test-Revised Delayed Recall (HVLT-R DL) were observed at 24 weeks (memantine median decline: 0 , placebo median decline: $-2, \mathrm{p}=0.059)$, participants treated with memantine showed delayed time to cognitive decline (HR 0.78, $95 \%$ CI $0.62-0.99, \mathrm{p}=0.01$ ) and superior executive function at 8 $(p=0.008)$ and 16 weeks $(p=0.0041)$, as well as superior processing speed $(\mathrm{p}=0.0137)$ and delayed recognition $(\mathrm{p}=0.0149)$ at 24 weeks (43). Following these results, the National Cancer Comprehensive Network (NCCN) incorporated the use of memantine along with WBRT into their consensus guidelines. Despite this, routine use of memantine for patients receiving WBRT has not yet been established; future health policy strategies should develop ways in which memantine can be more quickly adopted into routine therapy (83). Other agents, such as the donepezil, reninangiotensin system blockers, or Ginkgo biloba, have also been investigated (84-87).

The decline in memory function observed following WBRT may be a consequence of radiation-induced injury to the hippocampus, a region which is involved in neurocognitive functions, including memory, learning, and spatial information processing (88). Given the low frequency of $\mathrm{BrM}$ in the hippocampus, this region could represent a dose-limiting structure (89). Early data to support this hypothesis stems from a single-arm, multi-institutional phase II study (RTOG 0933) that showed superior cognitive preservation (as measured by the HVLT-R DR) with the use of hippocampal avoidance WBRT (HA-WBRT) compared with historical data from WBRT-treated controls (mean relative decline from baseline to 4 months $7 \%$ vs. $30 \%$, p <0.001) (45).

Given the success observed with memantine, a more recent phase III trial investigating the addition of memantine to HAWBRT showed significantly lower risk of cognitive failure after HAWBRT plus memantine compared with HA-WBRT alone (HR 0.74, 95\% CI 0.58-0.95) (90). Treatment efficacy in terms of intracranial PFS (iPFS) and OS did not differ between the two arms, suggesting that HA-WBRT plus memantine should be considered as standard of care for patients scheduled to receive WBRT with no $\mathrm{BrM}$ in the hippocampal region. As patients experience longer survival, neurological sequalae from radiation treatment will become increasingly important. Anatomical avoidance and pharmacotherapy are promising ways for clinicians to preserve cognitive function in patients receiving WBRT.

\subsubsection{Radiation Therapy in the Setting of SCLC}

WBRT does remain the standard of care for patients with SCLC in the form of prophylactic cranial irradiation (PCI). As many as $40-60 \%$ of patients with SCLC will develop IMD during the course of their disease, with rates of IMD 1.3-2 times higher than in patients with NSCLC (91). Notably, this standard is being challenged by mounting evidence from retrospective studies and meta-analyses on the lack of survival benefit in the extensive stage setting and additional neurotoxicity of PCI in patients with SCLC (92-94). As patients with SCLC have been historically excluded from trials for SRS, data comparing SRS and WRBT alone, or SRS and WBRT + boost, in this patient population is uniformly retrospective. A propensity score-matched observational study by Rusthoven et al. found that, as for other malignancies, the addition of WBRT to SRS improved intracranial control (time to central nervous system (CNS) progression HR $0.28, \mathrm{p}<0.001$ ), but not survival (median OS 5.2 months for WBRT vs. 6.5 months for SRS, $\mathrm{p}=0.79$ ), in patients with SCLC (95). Similarly, a phase III trial found equivalent $O S$ and intracranial control with WBRT with hippocampal sparing (HA-WBRT) compared to conventional WBRT, with prolonged preservation of neurocognitive function in the HA-WBRT arm (96). These findings suggest a trend away from conventional WBRT as PCI for patients with SCLC toward 
treatments aligned to achieve IMD control while maintaining QoL and cognition.

\subsection{Pharmacological Therapies for IMD}

Although surgery and radiation therapy remain the cornerstone of treatment for patients with IMD, recent data support a role for targeted systemic therapies and immunotherapy with immune check point inhibitors in certain patient subgroups.

\subsubsection{Targeted Therapies}

Advances in genetic and genomic analyses have enabled the discovery of genetic alterations that promote tumor growth and proliferation. In a subset of patients with breast cancer, for example, amplification of the human epidermal growth factor receptor 2 (HER2/neu) has been shown to drive cancer propagation (97-99). The development of small molecule or antibody-based agents to target these molecular drivers of cancer and their associated signalling pathways has revolutionized the treatment of patients with HER2-positive breast cancer, epidermal growth factor receptor (EGFR)-mutant or anaplastic lymphoma kinase (ALK)-rearranged NSCLC, and BRAF-mutant melanoma (Table 1).

\subsubsection{Targeted Therapies in Breast Cancer}

As IMD is a frequent complication in patients with breast cancer, a large body of literature is available that describes current efforts to identify and evaluate targeted therapy in HER2-positive breast cancer, hormone receptor (HR)-positive breast cancer, and triple negative breast cancer (TNBC).

2.3.1.1.1 HER2-Positive Breast Cancer. The addition of trastuzumab, a monoclonal antibody against the HER2 receptor, has been shown to prolong OS and PFS in patients with HER2positive breast cancer (100). While trastuzumab therapy has been demonstrated to improve systemic disease control, populationbased studies identified an increased incidence of IMD in patients treated with trastuzumab, likely resulting from the prolongation in survival and limited penetration of drug across the blood-brain barrier (BBB), rendering the brain a "sanctuary site" for cancer cells (150-152). Notably, HER2-positive breast cancer patients treated with palliative chemotherapy and trastuzumab were more likely to develop BrM than patients who received palliative chemotherapy alone $(37.8 \%$ vs. $25.0 \%$, $\mathrm{p}=0.028$ ); conversely, median time to death (TTD) measured from the development of IMD was significantly longer for patients treated with palliative chemotherapy and trastuzumab, compared to those treated with palliative chemotherapy alone (14.9 vs. 4.0 months, $\mathrm{p}=0.0005$ ), suggesting that trastuzumab might exert some biological effect, even if partial, on IMD (101). Supporting this hypothesis, studies have similarly found that trastuzumab prolongs median OS in HER2-positive breast cancer patients with IMD (102-104).

Lapatinib, an inhibitor of HER2 and EGFR, was subsequently developed to treat HER2-positive breast cancer that had progressed on all previous lines of therapy. From the perspective of IMD, lapatinib was theorized to have better BBB penetrance than trastuzumab, given its smaller molecular weight.
Initial phase II trial results in HER2-positive breast cancer patients with progressive IMD despite WBRT or SRS demonstrated modest antitumor activity with either lapatinib alone (CNS objective response rate (ORR): 2.6-6\%) or in combination with capecitabine (CNS ORR: 20\%) (105, 106). Subsequent work has suggested that the combination of lapatinib and capecitabine increases median OS from the time of IMD development in HER2-positive breast cancer patients previously treated with anthracycline, trastuzumab, and a taxane, compared with patients receiving anthracycline, trastuzumab, and a taxane only (27.9 vs. 16.7 months, $\mathrm{p}=0.01$ ) (107). The combination of lapatinib and capecitabine also demonstrated high antitumor activity (CNS ORR: 65.9\%) in HER2-positive breast cancer patients with previously untreated IMD in the LANDSCAPE trial, though the impact of treatment on OS was not explicitly determined and concerns regarding treatment toxicity and delays in accessing radiotherapy were raised (108).

Following the development of lapatinib, additional small molecule candidates have been engineered. Neratinib, an irreversible inhibitor of HER1, HER2, and HER4, has been demonstrated to have good antitumor activity in combination with capecitabine in lapatinib-naive or lapatinib-treated patients with IMD secondary to HER2-positive breast cancer (CNS ORR: $49 \%$ and $33 \%$, respectively). Among these two patient cohorts, median PFS was 5.5 and 3.1 months, respectively, and median OS was 13.3 and 15.1 months, respectively, though no direct comparisons between groups were reported (109). When compared head-to-head with lapatinib and capecitabine $(\mathrm{L}+\mathrm{C})$ in a recent RCT, neratinib and capecitabine $(\mathrm{N}+\mathrm{C})$ showed a substantial though not statistically significant effect on OS (13.9 vs. 2.4 months, HR $0.90, \mathrm{p}=0.635$ ) and PFS (5.6 vs. 4.3 months, HR 0.66, $\mathrm{p}=0.074$ ) in HER2-positive breast cancer patients with IMD who have previously failed at least two anti-HER2 therapies (110). The lack of statistical significance may have been due to small sample sizes. Of 621 patients enrolled, 101 (16.3\%) had known CNS metastases at baseline (N+C: $n=51$; $+\mathrm{C}: n=50)$; 81 had received prior CNS-directed radiotherapy or surgery. In the CNS subgroup, mean PFS through 24 months was 7.8 months with $\mathrm{N}+\mathrm{C}$ versus 5.5 months with $\mathrm{L}+\mathrm{C}$ (HR 0.66, 95\% CI 0.41-1.05), and mean OS through 48 months was 16.4 vs. 15.4 months (HR 0.90, 95\% CI 0.59-1.38). At 12 months, cumulative incidence of interventions for CNS disease was $25.5 \%$ for the $\mathrm{N}+\mathrm{C}$ group vs. $36.0 \%$ for the $\mathrm{L}+\mathrm{C}$ group, and cumulative incidence of progressive CNS disease was $26.2 \%$ versus $41.6 \%$, respectively. In patients with target CNS lesions at baseline $(n=32)$, confirmed intracranial ORR (iORR) were $26.3 \%$ and $15.4 \%$, respectively.

A third HER2 inhibitor, tucatinib, demonstrated excellent antitumor activity in combination with trastuzumab and capecitabine in HER2-positive breast cancer patients with IMD, compared to trastuzumab and capecitabine alone (iORR $47.3 \%$ vs. $20 \%, \mathrm{p}=0.03$ ), and prolonged median OS (18.1 vs. 12.0 months, HR 0.58, p=0.005) and median PFS (9.9 vs. 4.2 months, HR 0.32, p<0.0001) (111).

Multiple small studies have shown signal for intracranial activity in patients with metastatic HER2-positive breast cancer 
TABLE 1 | Summary of studies investigating targeted therapies for IMD secondary to breast cancer, NSCLC, and melanoma.

\begin{tabular}{|c|c|c|c|c|c|c|c|}
\hline & Drug & Trial/Study & Study design & $\begin{array}{l}\text { Total } \\
\text { participants } \\
\text { (n) }\end{array}$ & Study arms & $\begin{array}{l}\text { Median OS } \\
\text { (months) }\end{array}$ & Findings \\
\hline \multirow[t]{28}{*}{$\begin{array}{l}\text { Breast } \\
\text { cancer }\end{array}$} & \multirow[t]{7}{*}{ Trastuzumab } & $\begin{array}{l}\text { Slamon } \\
\text { et al. (100) }\end{array}$ & $\mathrm{RCT}$ & 469 & $\begin{array}{l}\text { Standard chemotherapy } \pm \\
\text { trastuzumab in all breast } \\
\text { cancer patients }\end{array}$ & $\begin{array}{l}25.1 \text { vs. } 20.3 \\
(p=0.046)\end{array}$ & $\begin{array}{l}\text { Relative risk reduction of death at } 30 \text { - } \\
\text { month follow-up: } 20 \%\end{array}$ \\
\hline & & $\begin{array}{l}\text { Park et al. } \\
(101)\end{array}$ & $\begin{array}{l}\text { Retrospective } \\
\text { cohort study }\end{array}$ & 251 & $\begin{array}{l}\text { Palliative chemotherapy } \pm \\
\text { trastuzumab in all breast }\end{array}$ & $\begin{array}{l}31.7 \text { vs. } 16.7 \\
(p=0.001)\end{array}$ & $\begin{array}{l}\text { Incidence of BrM: } 37.8 \text { vs. } 25 \% \\
(p=0.028)\end{array}$ \\
\hline & & & & & cancer patients & & $\begin{array}{l}\text { TTD from BrM: } 14.9 \text { vs. } 4.0 \text { months } \\
(p=0.0005)\end{array}$ \\
\hline & & $\begin{array}{l}\text { Park et al. } \\
(102)\end{array}$ & $\begin{array}{l}\text { Retrospective } \\
\text { cohort study }\end{array}$ & 78 & $\begin{array}{l}\text { Trastuzumab after BrM } \\
\text { diagnosis vs. trastuzumab }\end{array}$ & $\begin{array}{l}13.6 \text { vs. } 5.5 \text { vs. } \\
4.0(p<0.001)\end{array}$ & $\begin{array}{l}\text { Median TTP of BrM: } 7.8 \text { vs. } 3.9 \text { vs. } 2.9 \\
\text { months }(p=0.006)\end{array}$ \\
\hline & & & & & $\begin{array}{l}\text { before BrM diagnosis only } \\
\text { vs. no trastuzumab }\end{array}$ & & $\begin{array}{l}\text { HR for death in patients with BrM: } 0.5 \\
(p=0.017)\end{array}$ \\
\hline & & $\begin{array}{l}\text { Okita et al. } \\
(103)\end{array}$ & $\begin{array}{l}\text { Retrospective } \\
\text { cohort study }\end{array}$ & 62 & $\begin{array}{l}\text { Trastuzumab vs. no } \\
\text { trastuzumab }\end{array}$ & $\begin{array}{l}38.4 \text { vs } 8.4 \\
(p=0.0005)\end{array}$ & $\begin{array}{l}\text { Median second brain metastatic-free } \\
\text { survival time: } 7.0 \text { vs. } 5.6 \text { months } \\
(p=0.057)\end{array}$ \\
\hline & & $\begin{array}{l}\text { Dawood } \\
\text { et al. (104) }\end{array}$ & $\begin{array}{l}\text { Retrospective } \\
\text { cohort study }\end{array}$ & 598 & $\begin{array}{l}\text { Trastuzumab vs. no } \\
\text { trastuzumab vs. HER2- } \\
\text { negative }\end{array}$ & $\begin{array}{l}11.6 \text { vs. } 6.1 \text { vs. } \\
6.3(p<0.0001)\end{array}$ & - \\
\hline & \multirow[t]{6}{*}{ Lapatinib } & $\begin{array}{l}\text { Lin et al. } \\
(105)\end{array}$ & $\begin{array}{l}\text { Single-arm } \\
\text { clinical trial }\end{array}$ & 39 & Lapatinib & - & CNS ORR: $2.6 \%$ \\
\hline & & $\begin{array}{l}\text { Lin et al. } \\
(106)\end{array}$ & $\begin{array}{l}\text { Single-arm } \\
\text { clinical trial }\end{array}$ & 242 & $\begin{array}{l}\text { Lapatinib, lapatinib and } \\
\text { capecitabine }(n=50)\end{array}$ & - & $\begin{array}{l}\text { CNS ORR: } 6 \% \text { (lapatinib alone), } 20 \% \\
\text { (with capecitabine) } \\
\geq 20 \% \text { BrM volume reduction: } 21 \% \\
\text { (lapatinib alone), } 40 \% \text { (with capecitabine) }\end{array}$ \\
\hline & & Metro et al. & Retrospective & 30 & Lapatinib and & 27.9 vs. 16.7 & CNS ORR: $31.8 \%$ \\
\hline & & $(107)$ & cohort study & & capecitabine & $(p=0.01)$ & Disease stabilization: $27.3 \%$ \\
\hline & & $\begin{array}{l}\text { Bachelot } \\
\text { et al. (108) }\end{array}$ & $\begin{array}{l}\text { Single-arm } \\
\text { clinical trial }\end{array}$ & 45 & $\begin{array}{l}\text { Lapatinib and } \\
\text { capecitabine }\end{array}$ & 17.0 & $\begin{array}{l}\text { Median TTP: } 5.5 \text { months } \\
\text { CNS ORR: } 65.9 \%\end{array}$ \\
\hline & & & & & & & Disease stabilization: $36 \%$ \\
\hline & \multirow[t]{2}{*}{ Neratinib } & $\begin{array}{l}\text { Freedman } \\
\text { et al. (109) }\end{array}$ & $\begin{array}{l}\text { Single-arm } \\
\text { clinical trial }\end{array}$ & 49 & $\begin{array}{l}\text { Neratinib and capecitabine } \\
\text { in lapatinib-naïve and } \\
\text { lapatinib-treated patients }\end{array}$ & 13.3 and 15.1 & $\begin{array}{l}\text { CNS ORR: } 49 \% \text { and } 33 \% \\
\text { Median PFS: } 5.5 \text { and } 3.1 \text { months }\end{array}$ \\
\hline & & $\begin{array}{l}\text { Hurvitz et al. } \\
(110)\end{array}$ & RCT & 101 & $\begin{array}{l}\text { Neratinib and capecitabine } \\
\text { vs. lapatinib and } \\
\text { capecitabine }\end{array}$ & $\begin{array}{l}13.9 \text { vs. } 12.4 \\
(p=0.635)\end{array}$ & $\begin{array}{l}\text { Median PFS: } 5.6 \text { vs. } 4.3 \text { months } \\
(p=0.074)\end{array}$ \\
\hline & \multirow[t]{2}{*}{ Tucatinib } & \multirow[t]{2}{*}{$\begin{array}{l}\text { Lin et al. } \\
(111)\end{array}$} & \multirow[t]{2}{*}{$\mathrm{RCT}$} & \multirow[t]{2}{*}{291} & \multirow{2}{*}{$\begin{array}{l}\text { Trastuzumab and } \\
\text { capecitabine with or } \\
\text { without tucatinib }\end{array}$} & \multirow[t]{2}{*}{$\begin{array}{l}18.1 \text { vs. } 12.0 \\
(p=0.005)\end{array}$} & $\begin{array}{l}\text { Median PFS: } 9.9 \text { vs. } 4.2 \text { months, HR } \\
0.32, P<0.0001\end{array}$ \\
\hline & & & & & & & iORR: $47.3 \%$ vs. $20 \%, p=0.03$ \\
\hline & $\begin{array}{l}\text { Trastuzumab } \\
\text { emtansine }\end{array}$ & $\begin{array}{l}\text { Bartsch } \\
\text { et al. (112) }\end{array}$ & $\begin{array}{l}\text { Retrospective } \\
\text { cohort study }\end{array}$ & 10 & $\mathrm{~T}-\mathrm{DM} 1$ & - & iORR: $30 \%$ \\
\hline & \multirow[t]{3}{*}{$(\mathrm{T}-\mathrm{DM} 1)$} & $\begin{array}{l}\text { Jacot et al. } \\
(113)\end{array}$ & $\begin{array}{l}\text { Single-arm } \\
\text { clinical trial }\end{array}$ & 2002 & T-DM1 & - & - \\
\hline & & $\begin{array}{l}\text { Krop et al. } \\
(114)\end{array}$ & $\mathrm{RCT}$ & 991 & $\begin{array}{l}\text { T-DM1 vs. capecitabine } \\
\text { and laptinib }\end{array}$ & $\begin{array}{l}26.8 \text { vs. } 12.9 \\
(H R 0.38 \\
p=0.008)\end{array}$ & $\begin{array}{l}\text { Median PFS: } 5.9 \text { vs. } 5.7 \text { months (HR } \\
1.00, p=1.0)\end{array}$ \\
\hline & & $\begin{array}{l}\text { Montemurro } \\
\text { et al. (115) }\end{array}$ & $\begin{array}{l}\text { Single-arm } \\
\text { clinical trial }\end{array}$ & 2002 & T-DM1 & 18.9 & $\begin{array}{l}\text { Median PFS: } 5.5 \text { months } \\
\text { ORR: } 21.4 \%\end{array}$ \\
\hline & $\begin{array}{l}\text { Trastuzumab } \\
\text { deruxtecan (T- }\end{array}$ & $\begin{array}{l}\text { Barsch et al. } \\
(116)\end{array}$ & $\begin{array}{l}\text { Single-arm } \\
\text { clinical trial }\end{array}$ & 10 & T-DXd & - & CNS ORR: $83.3 \%$ \\
\hline & $\mathrm{DXd})$ & $\begin{array}{l}\text { Jerusalem } \\
\text { et al. (117) }\end{array}$ & $\begin{array}{l}\text { Single-arm } \\
\text { clinical trial }\end{array}$ & 24 & T-DXd & - & $\begin{array}{l}\text { Median PFS: } 18.1 \text { months } \\
\text { ORR: } 58.3 \% \\
\text { CNS ORR: } 50 \%\end{array}$ \\
\hline & \multirow[t]{2}{*}{ Abemaciclib } & \multirow[t]{2}{*}{$\begin{array}{l}\text { Tolaney } \\
\text { et al. (118) }\end{array}$} & $\begin{array}{l}\text { Non- } \\
\text { randomized }\end{array}$ & 104 & $\begin{array}{l}\text { Abemaciclib } \pm \text { hormone } \\
\text { therapy }\end{array}$ & 12.5 & CNS ORR: $5.2 \%$ \\
\hline & & & clinical trial & & $\begin{array}{l}\text { Abemaciclib and } \\
\text { trastuzumab }\end{array}$ & 10.1 & $\begin{array}{l}\text { CNS ORR: 0\% } \\
\text { Median intracranial PFS: } 2.7 \text { months }\end{array}$ \\
\hline & Palbociclib & $\begin{array}{l}\text { Brastianos } \\
\text { et al. (119) }\end{array}$ & $\begin{array}{l}\text { Single-arm } \\
\text { clinical trial }\end{array}$ & 15 & Palbociclib & 6.4 & Intracranial disease benefit rate: 53.3\% \\
\hline & Iniparib & $\begin{array}{l}\text { Anders et al. } \\
(120)\end{array}$ & $\begin{array}{l}\text { Single-arm } \\
\text { clinical trial }\end{array}$ & 37 & Iniparib and irinotecan & 7.83 & CNS ORR: $12 \%$ \\
\hline & Talazoparib & $\begin{array}{l}\text { Litton et al. } \\
(121)\end{array}$ & $\mathrm{RCT}$ & 431 & $\begin{array}{l}\text { Talazoparib vs. } \\
\text { chemotherapy }\end{array}$ & & - \\
\hline
\end{tabular}


TABLE 1 | Continued

\begin{tabular}{|c|c|c|c|c|c|c|c|}
\hline & Drug & Trial/Study & Study design & $\begin{array}{l}\text { Total } \\
\text { participants } \\
\text { (n) }\end{array}$ & Study arms & $\begin{array}{l}\text { Median OS } \\
\text { (months) }\end{array}$ & Findings \\
\hline & & & & & & $\begin{array}{l}- \\
\text { (HR } 0.671,95 \% \\
\text { Cl } 0.366-1.229)\end{array}$ & \\
\hline \multirow[t]{22}{*}{ NSCLC } & Crizotinib & $\begin{array}{l}\text { Solomon } \\
\text { et al. (122) }\end{array}$ & RCT & 343 & $\begin{array}{l}\text { Crizotinib vs. pemetrexed } \\
+ \text { platinum-based } \\
\text { chemotherapy }\end{array}$ & - & $\begin{array}{l}\text { Median PFS: } 9.0 \text { vs. } 4.0 \text { months, HR } \\
0.40, P<0.001 \\
\text { iORR: } 77 \% \text { vs. } 28 \%, p<0.001\end{array}$ \\
\hline & Ceritinib & $\begin{array}{l}\text { Crinò et al. } \\
(123)\end{array}$ & $\begin{array}{l}\text { Single-arm } \\
\text { clinical trial }\end{array}$ & 140 & Ceritinib & - & $\begin{array}{l}\text { Median PFS: } 5.4 \text { months } \\
\text { Intracranial ORR: } 33 \%\end{array}$ \\
\hline & Alectinib & $\begin{array}{l}\text { Gadgeel } \\
\text { et al. (124) }\end{array}$ & $\begin{array}{l}\text { Single-arm } \\
\text { clinical trial }\end{array}$ & 47 & Alectinib & - & iORR: $52 \%$ \\
\hline & & $\begin{array}{l}\text { Peters et al. } \\
(125)\end{array}$ & $\mathrm{RCT}$ & 303 & Alectinib vs. crizotinib & - & $\begin{array}{l}\text { PFS rate: } 12 \% \text { vs. } 45 \%, \text { HR } 0.51 \\
p<0.001\end{array}$ \\
\hline & Brigatinib & $\begin{array}{l}\text { Camidge } \\
\text { et al. (126) }\end{array}$ & $\mathrm{RCT}$ & 275 & Brigatinib vs. crizotinib & - & $\begin{array}{l}\text { 12-month PFS rate: } 67 \% \text { vs. } 21 \% \text {, HR } \\
0.27 \\
\text { Intracranial median TTP: HR } 0.30 \\
\text { iORR: } 78 \% \text { vs. } 29 \% \text {, OR } 10.42\end{array}$ \\
\hline & Lorlatinib & $\begin{array}{l}\text { Shaw et al. } \\
(127)\end{array}$ & $\mathrm{RCT}$ & 296 & Lorlatinib vs. crizotinib & - & $\begin{array}{l}\text { 12-month PFS rate: } 96 \% \text { vs. } 60 \% \text {, HR } \\
0.07 \\
\text { iORR: } 82 \% \text { vs. } 23 \% \text {, OR } 16.83\end{array}$ \\
\hline & & $\begin{array}{l}\text { Shaw et al. } \\
(128)\end{array}$ & $\begin{array}{l}\text { Single-arm } \\
\text { clinical trial }\end{array}$ & 364 & Lorlatinib & - & $\begin{array}{l}\text { CNS ORR (TKI-naive): } 64 \% \\
\text { CNS ORR (previous crizotinib): } 50 \%\end{array}$ \\
\hline & Ensartinib & $\begin{array}{l}\text { Horn et al. } \\
\text { (129) }\end{array}$ & RCT & 290 & Ensartinib vs. crizotinib & - & $\begin{array}{l}\text { Median PFS (baseline BrM): } \\
11.8 \text { vs. } 7.5 \text { months (HR 0.55, } p=0.05 \text { ) } \\
\text { Median PFS (no baseline BrM): } \\
\text { NR vs. } 16.6 \text { months (HR } 0.46, p=0.003 \text { ) }\end{array}$ \\
\hline & Lazertinib & $\begin{array}{l}\text { Ahn et al. } \\
(130)\end{array}$ & $\begin{array}{l}\text { Single-arm } \\
\text { clinical trial }\end{array}$ & 127 & Lazertinib & - & CNS ORR: $44 \%$ \\
\hline & & $\begin{array}{l}\text { Cho et al. } \\
(131)\end{array}$ & $\begin{array}{l}\text { Single-arm } \\
\text { clinical trial }\end{array}$ & 78 & Lazertinib & - & CNS ORR: $85.7 \%$ \\
\hline & Furmonertinib & $\begin{array}{l}\text { Shi et al. } \\
(132)\end{array}$ & $\begin{array}{l}\text { Single-arm } \\
\text { clinical trial }\end{array}$ & 130 & Furmonertinib & - & $\begin{array}{l}\text { Median PFS: } 9.9 \text { months } \\
\text { CNS ORR: } 58.8 \%\end{array}$ \\
\hline & & $\begin{array}{l}\text { Shi et al. } \\
(133)\end{array}$ & $\begin{array}{l}\text { Single-arm } \\
\text { clinical trial }\end{array}$ & 220 & Furmonertinib & - & $\begin{array}{l}\text { CNS ORR (measurable BrM): } 66 \% \\
\text { CNS ORR (measurable/non-measurable } \\
\text { BrM): } 34 \% \\
\text { Median PFS (measurable/non- } \\
\text { measurable BrM): } 11.6 \text { months }\end{array}$ \\
\hline & Amivantamab & $\begin{array}{l}\text { Park et al. } \\
\text { (134) }\end{array}$ & $\begin{array}{l}\text { Single-arm } \\
\text { clinical trial }\end{array}$ & 81 & Amivantamab & - & ORR: $39 \%$ \\
\hline & Gefitinib & $\begin{array}{l}\text { Ceresoli } \\
\text { et al. (135) }\end{array}$ & $\begin{array}{l}\text { Single-arm } \\
\text { clinical trial }\end{array}$ & 41 & Gefitinib & - & $\begin{array}{l}\text { Median PFS: } 3.0 \text { months } \\
\text { iORR: } 10 \% \\
\text { DCR: } 27 \%\end{array}$ \\
\hline & & $\begin{array}{l}\text { Hotta et al. } \\
(136)\end{array}$ & $\begin{array}{l}\text { Retrospective } \\
\text { cohort study }\end{array}$ & 57 & Gefitinib & - & iORR: $42.9 \%$ \\
\hline & & $\begin{array}{l}\text { Lee et al. } \\
\text { (137) }\end{array}$ & $\begin{array}{l}\text { Single-arm } \\
\text { clinical trial }\end{array}$ & 37 & Gefitinib & - & iORR: $70 \%$ \\
\hline & & $\begin{array}{l}\text { Chiu et al. } \\
(138)\end{array}$ & $\begin{array}{l}\text { Single-arm } \\
\text { clinical trial }\end{array}$ & 76 & Gefitinib & - & $\begin{array}{l}\text { iORR: } 33.3 \% \\
\text { DCR: } 63.2 \%\end{array}$ \\
\hline & & $\begin{array}{l}\text { Kim et al. } \\
(139)\end{array}$ & $\begin{array}{l}\text { Double-arm } \\
\text { clinical trial }\end{array}$ & 23 & Gefitinib or erlotinib & 18.8 & $\begin{array}{l}\text { Median PFS: } 7.1 \text { months } \\
\text { iORR: } 73.9 \% \\
\text { Overall ORR: } 69.6 \% \\
\text { Overall DCR: } 82.6 \%\end{array}$ \\
\hline & & $\begin{array}{l}\text { Park et al. } \\
(140)\end{array}$ & $\begin{array}{l}\text { Double-arm } \\
\text { clinical trial }\end{array}$ & 28 & Gefitinib or erlotinib & 15.9 & $\begin{array}{l}\text { Median PFS: } 6.6 \text { months } \\
\text { Overall ORR: } 83 \% \\
\text { Overall DCR: } 93 \%\end{array}$ \\
\hline & Osimertinib & $\begin{array}{l}\text { Mok et al. } \\
(141)\end{array}$ & $\mathrm{RCT}$ & 419 & $\begin{array}{l}\text { Osimertinib vs. } \\
\text { pemetrexed with platinum- } \\
\text { based chemotherapy }\end{array}$ & - & $\begin{array}{l}\text { Median PFS: } 8.5 \text { vs. } 4.2 \text { months, HR } \\
0.32\end{array}$ \\
\hline & & $\begin{array}{l}\text { Soria et al. } \\
(142)\end{array}$ & $\mathrm{RCT}$ & 456 & $\begin{array}{l}\text { Osimertinib vs. erlotinib or } \\
\text { gefitinib }\end{array}$ & - & $\begin{array}{l}\text { Median PFS: } 15.2 \text { vs. } 9.6 \text { months, HR } \\
0.47, p<0.001\end{array}$ \\
\hline & Sotorasib & $\begin{array}{l}\text { Skoulidis } \\
\text { et al. (143) }\end{array}$ & $\begin{array}{l}\text { Single-arm } \\
\text { clinical trial }\end{array}$ & 126 & $\begin{array}{l}\text { Sotorasib in all KRAS }{ }^{\mathrm{G} 12 \mathrm{C}_{-}} \\
\text {positive patients }\end{array}$ & 12.5 & $\begin{array}{l}\text { Median PFS: } 6.8 \text { months } \\
\text { Overall ORR: } 37.1 \%\end{array}$ \\
\hline
\end{tabular}

(Continued) 
TABLE 1 | Continued

\begin{tabular}{|c|c|c|c|c|c|c|c|}
\hline & Drug & Trial/Study & Study design & $\begin{array}{l}\text { Total } \\
\text { participants } \\
\text { (n) }\end{array}$ & Study arms & $\begin{array}{l}\text { Median OS } \\
\text { (months) }\end{array}$ & Findings \\
\hline & Selpercatinib & $\begin{array}{l}\text { Drilon et al. } \\
(144)\end{array}$ & $\begin{array}{l}\text { Single-arm } \\
\text { clinical trial }\end{array}$ & 105 & Selpercatinib & - & CNS ORR: 91\% \\
\hline & Pralsetinib & $\begin{array}{l}\text { Gainor et al. } \\
(145)\end{array}$ & $\begin{array}{l}\text { Single-arm } \\
\text { clinical trial }\end{array}$ & 233 & Pralsetinib & - & CNS ORR: $56 \%$ \\
\hline & Repotrectinib & $\begin{array}{l}\text { Drilon et al. } \\
(146)\end{array}$ & $\begin{array}{l}\text { Single-arm } \\
\text { clinical trial }\end{array}$ & - & Repotrectinib & - & - \\
\hline & Tepotinib & Paik et al. & $\begin{array}{l}\text { Single-arm } \\
\text { clinical trial }\end{array}$ & 152 & Tepotinib & - & $\begin{array}{l}\text { Median PFS: } 10.0 \text { months } \\
\text { ORR: } 55 \%\end{array}$ \\
\hline & Capmatinib & Wolf et al. & $\begin{array}{l}\text { Single-arm } \\
\text { clinical trial }\end{array}$ & 364 & Capmatinib & - & CNS ORR: $53.8 \%$ \\
\hline & Laprotrectinib & Hong et al. & $\begin{array}{l}\text { Single-arm } \\
\text { clinical trial }\end{array}$ & 159 & Laprotrectinib & - & CNS ORR: $66.7 \%$ \\
\hline & Entrectinib & John et al. & $\begin{array}{l}\text { Single-arm } \\
\text { clinical trial }\end{array}$ & 16 & Entrectinib & - & $\begin{array}{l}\text { CNS ORR (measurable BrM): } 62.5 \% \\
\text { CNS ORR (measurable/non-measurable } \\
\text { BrM): } 50 \%\end{array}$ \\
\hline \multirow[t]{3}{*}{ Melanoma } & Dabrafenib & $\begin{array}{l}\text { Long et al. } \\
(147)\end{array}$ & $\begin{array}{l}\text { Single-arm } \\
\text { clinical trial }\end{array}$ & 172 & $\begin{array}{l}\text { Dabrafenib in BRAF }{ }^{\mathrm{V} 600 E_{-}} \\
\text {positive melanoma } \\
\text { patients with treatment- } \\
\text { naïve IMD or progressive } \\
\text { IMD }\end{array}$ & 7.64 and 7.25 & $\begin{array}{l}\text { Median PFS: } 3.72 \text { and } 3.83 \text { months } \\
\text { iORR: } 39.2 \% \text { and } 30.8 \%\end{array}$ \\
\hline & & $\begin{array}{l}\text { Davies et al. } \\
(148)\end{array}$ & $\begin{array}{l}\text { Single-arm } \\
\text { clinical trial }\end{array}$ & 125 & $\begin{array}{l}\text { Dabrafenib and trametinib } \\
\text { in BRAF }{ }^{\mathrm{V} 600 E_{-}} \text {-positive } \\
\text { melanoma patients with } \\
\text { treatment-naïv IMD or } \\
\text { progressive IMD }\end{array}$ & 10.8 and 24.3 & $\begin{array}{l}\text { Median PFS: } 5.6 \text { and } 7.2 \text { months } \\
\text { iORR: } 58 \% \text { and } 56 \%\end{array}$ \\
\hline & Vemurafenib & $\begin{array}{l}\text { McArthur } \\
\text { et al. (149) }\end{array}$ & $\begin{array}{l}\text { Single-arm } \\
\text { clinical trial }\end{array}$ & 146 & $\begin{array}{l}\text { Vemurafenib in BRAF }{ }^{\mathrm{V} 600} \text { - } \\
\text { positive melanoma } \\
\text { patients with treatment- } \\
\text { naïve IMD or progressive } \\
\text { IMD }\end{array}$ & 8.9 and 9.6 & $\begin{array}{l}\text { Median PFS: } 3.7 \text { and } 4.0 \text { months } \\
\text { iORR: } 18 \% \text { and } 18 \%\end{array}$ \\
\hline
\end{tabular}

Median overall survival marked with a dash if the data was 1) not reported or 2) reported for the entire population, including patients without IMD.

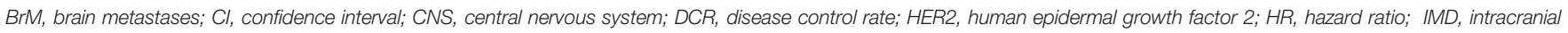

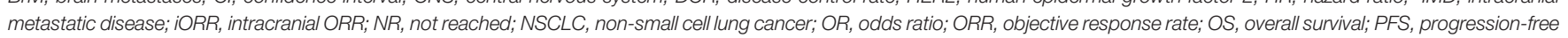
survival; RCT, randomized control trial; T-DM1, trastuzumab emtansine; T-DXd, trastuzumab deruxtecan; TKI, tyrosine kinase inhibitor; TTD, time to death; TTP, time to progression.

with the antibody-drug conjugate trastuzumab emtansine (TDM1) (112, 113); secondary analysis has further shown T-DM1 to improve OS in patients with trastuzumab-resistant advanced metastatic breast cancer and asymptomatic BrM previously treated with radiotherapy, compared with lapatinib plus capecitabine (114). An exploratory final analysis of the ongoing KAMILLA trial, an international, single-arm, open-label, phase IIIb study evaluating the safety and efficacy of T-DM1 in patients with previously treated, HER2-positive advanced breast cancer, showed a high CNS-specific ORR, including a CNS-specific ORR of $\sim 50 \%$ in a subgroup of 67 patients who had not received prior radiation therapy for BrM (115).

Similar to T-DM1, trastuzumab deruxtecan (T-DXd) is an antibody-drug conjugate that has been shown to demonstrate potential therapeutic benefits for HER2-positive breast cancer patients with BrM in early analyses of multiple phase II/III trials, although these analyses have only been reported in abstracts. For example, a preliminary analysis of an ongoing phase II trial, TUXEDO-1, demonstrated an initial iORR of $83.3 \%$ in participants enrolled in the first stage of the study (116), while a subgroup analysis of DESTINY-Breast01, another currently active phase II study, found an ORR of 58.3\% and median PFS of 18.1 months (95\% CI 6.7-18.1) in 24 patients treated with T-DXd (117). Encouraging results were recently presented by Hurvitz and colleagues in a subgroup analysis of DESTINY-Breast03 (NCT03529110), an ongoing phase III trial, comparing T-DXd and T-DM1 in HER2-positive breast cancer patients previously treated with trastuzumab and taxane-based chemotherapy, and we eagerly await publication of their abstract/full-text article.

While the availability of multiple treatment regimens consisting of single and combination agents has broadened the treatment of HER2-positive breast cancer with BrM, most studies in this area have historically excluded IMD patients. In addition to the identification and validation of new agents, current efforts are aimed at understanding the therapeutic efficacy and toxicities of these targeted therapies, and future studies should focus on the possibility of combining these agents with brain-directed radiation therapies to treat BrM.

2.3.1.1.2 Hormone Receptor-Positive Breast Cancer. HR-positive, HER2-negative breast cancers represent a subtype of breast cancers that do not respond to HER2 inhibitors, such as 
trastuzumab. Historically, treatment of HR-positive breast cancer has been limited to hormonal therapies, including aromatase inhibitors, selective estrogen receptor modulators, and estrogen receptor downregulators. Recent efforts in drug development have identified three cyclin-dependent kinase 4/6 (CDK4/6) inhibitors to enhance the management of HR-positive breast cancer. However, the landmark trials investigating CDK 4/6 inhibitors, such MONARCH 1-3, MONALEESA-2, and PALOMA-1, have excluded patients with $\operatorname{BrM}(153,154)$. Among HR-positive, HER2-negative breast cancer patients with BrM, abemaciclib demonstrated an iORR of $5.2 \%$ and median OS of 12.5 months (95\% CI 9.3-16.4) in one phase II trial (118). In the same trial, the combination of abemaciclib and trastuzumab did not demonstrate objective intracranial responses in patients with HR-positive, HER2-positive breast cancer. Patients who received abemaciclub and trastuzumab had a median OS of 10.1 months (95\% 4.2-14.3) and median iPFS of 2.7 months (95\% CI 1.4-4.0) (118). A second CDK4/6 inhibitor, palbociclib, demonstrated an intracranial benefit rate of $53.3 \%$ and median OS of 6.4 months (90\% CI 2.8-6.8) in a small prospective trial of patients with BrM secondary to breast cancer, melanoma, NSCLC, and esophageal cancer (119). Specific outcomes of the $3 / 15$ patients with HR-positive, HER2-negative breast cancer were not reported, limiting our ability to draw conclusions on the effectiveness of palbociclib in patients with HR-positive, HER2-negative breast cancer. Several clinical trials have been launched to investigate the role of CDK4/6 inhibitors in patients with HR-positive breast cancer patients with $\mathrm{BrM}$ (NCT04791384, NCT04923542, and NCT04227327).

2.3.1.1.3 Triple-Negative Breast Cancer. Approximately $50 \%$ of patients with TNBC are diagnosed with BrM (155). In this patient population, the survival prognosis following BrM diagnosis remains guarded given the lack of molecular targets compared with HER2-positive and HR-positive breast cancer. Poly (ADP-ribose) polymerase (PARP) inhibitors have been investigated in TNBC patients with BrM. The combination of the PARP inhibitor iniparib and the anti-cancer agent irinotecan demonstrated an iORR of $12.0 \%$ and median OS of 7.83 months $(95 \%$ CI 5.10-10.2) (120). A second PARP inhibitor talazoparib was evaluated in the phase III EMBRACA trial and did not significantly prolong OS in a subgroup of TNBC patients with a history of BrM (HR 0.671, 95\% CI 0.366-1.229) (121). Given treatment challenges with targeted therapies, there is a trend toward immunotherapies in TNBC, which will be discussed in the appropriate section of this review.

\subsubsection{Targeted Therapies in NSCLC}

IMD is a frequent complication of NSCLC. Here, we discuss current targeted therapy efforts for three common genetic alterations associated with NSCLC: ALK, EGFR, and Kirsten rat sarcoma virus (KRAS).

2.3.1.2.1 ALK Rearrangements in NSCLC. BrM have been reported to occur in $15-35 \%$ of patients with ALK-positive NSCLC, and up to $60 \%$ of these patients develop IMD after firstline therapy (156). In the PROFILE 104 trial, the first-generation
ALK inhibitor crizotinib improved median PFS in patients with ALK-positive NSCLC with IMD when administered as a single agent compared with platinum-based chemotherapy (9.0 vs. 4.0 months, HR 0.40, p<0.001), however, likely due to low CNS penetration of crizotinib, approximately half of patients suffered CNS progression (122). First line treatment for ALK-positive NSCLC has therefore shifted to next generation ALK TKIs that offer longer PFS and have greater activity within the CNS. In the ASCEND-2 trial, 100/140 enrolled ALK-positive NSCLC patients with IMD and previously treated with crizotinib and another regimen, received ceritinib (123). Of these patients, 33\% achieved an ORR with a median PFS of 5.4 months. The median OS for this subset of patients with IMD was not reported (123). Similarly, alectinib was found to demonstrate adequate antitumor activity in 52\% of crizotinib-resistant, ALK-positive NSCLC patients with IMD in an early single-arm phase II trial (124).

These findings were supported by the ALEX trial, which demonstrated IMD progression in $12 \%$ of untreated ALKpositive NSCLC patients with IMD receiving alectinib compared with $45 \%$ of patients receiving crozitinb (HR 0.51 , $\mathrm{p}<0.001$ ) (125). In ALK-positive NSCLC patients with measurable IMD and no previous ALK inhibitor treatment, patients randomized to treatment with brigatinib demonstrated an iORR of $78 \%$ compared with an iORR of $29 \%$ in patients randomized to receive crizotinib (OR 10.42) (126). Collectively, these results suggest that second generation ALK inhibitors effectively reduce IMD progression and death in patients with IMD secondary to ALK-positive NSCLC, compared with crizotinib.

The recent CROWN RCT investigated the third generation ALK inhibitor lorlatinib in ALK-positive NSCLC patients with measurable IMD and no prior systemic therapies. Adequate intracranial anti-tumor activity was reported in $82 \%$ of patients receiving lorlatinib compared with $23 \%$ of patients receiving crizotinib (OR 16.83) (127). The 12-month iPFS rate among patients treated with lorlatinib was $96 \%$, compared with $60 \%$ in patients treated with crizotinib (HR 0.07, 95\% CI 0.03-0.117) (127). Ensartinib, another ALK-inhibitor, has also been demonstrated to have high therapeutic efficacy among patients with ALK-positive NSCLC and BrM in the eXalt3 trial (129). Among patients with baseline BrM, those treated with ensartinib had higher median PFS compared to those treated with crizotinib (11.8 vs. 7.5 months, HR 0.55, 95\% 0.30-1.01, p=0.05). A similar trend in median PFS was observed among patients without baseline BrM receiving ersartinib versus crizotinib (NR vs. 16.6 months, HR 0.46, 95\% CI 0.27-0.77, p=0.003). Of note, the incidence of BrM was lower in patients receiving ersantinib compared with those receiving crozitinib at 12-months followup (cause-specific HR 0.32, 95\% 0.16-0.63, p=0.001) (129). These findings suggest that ALK inhibitors may be effective as monotherapies in patients with ALK-positive NSCLC patients and IMD.

Notably, these trials were all designed with intent to allow for study of drug effect on IMD. First, the trials described above allowed entry of patients that had untreated asymptomatic BrM. 
Second, all trials required that patient undergo MRI of the brain at accrual, regardless of IMD status, then mandated routine surveillance MRI while patients remained on trial. This approach allowed investigators to measure "prevention" of BrM. Finally, all three trials were compared the study drug to crizotinib, a systemically effective agent with poor CNS activity. This design could serve as a model for future trials designed to include the study of intracranial disease.

2.3.1.2.2 EGFR Mutations in NSCLC. Activating mutations in EGFR are present in $14-47 \%$ of NSCLC cases, and $2-63 \%$ of patients with EGFR-mutant NSCLC develop IMD during the course of their disease, accounting for a significant proportion of NSCLC-IMD cases, especially in East Asian populations (157). The first generation EGFR inhibitors, erlotinib and gefitinib, demonstrated increased efficacy compared with systemic chemotherapy in patients with EGFR-mutant NSCLC (157). Among patients with IMD secondary to EGFR-mutant NSCLC and previous chemotherapy or WBRT, treatment with gefitinib resulted in a partial response in about $10 \%$ of patients, and the overall disease control rate and median PFS were found to be $27 \%$ and 3 months, respectively (135). Similar results were obtained in subsequent single-arm clinical trials (136-138). Noncomparative trials with EGFR-mutant NSCLC patients with IMD receiving erlotinib or gefitinib as first-line therapy report an iORR of $73.9 \%$, median OS of 18.8 months, and PFS of 7.1 months (139). These results have since been reproduced with no statistically significant differences in OS found between erlotinib and gefitinib (140). The second-generation agent, afatinib, has shown similarly low activity in the CNS.

Of more clinical relevance is the third-generation agent, osimertinib, given its high efficacy in treatment-resistant, EGFR-mutant NSCLC and high CNS activity (158). In EGFRmutant NSCLC patients with IMD and known or likely resistance to first- and second-generation EGFR inhibitors, osimertinib was found to be more effective than pemetrexedbased chemotherapy regimens (median PFS 8.5 vs. 4.2 months, HR 0.32, 95\% CI 0.21-0.49) (141). The benefits of osimertinib were more pronounced in untreated patients with EGFR-mutant NSCLC and IMD when compared with the first-generation EGFR inhibitors erlotinib and gefitinib (median PFS 15.2 vs. 9.6 months, HR 0.47, p<0.001) (142). Given its effectiveness in delaying intracranial progression and death, osimeritinib has become the first-line treatment for patients with EGFR-mutant NSCLC (159).

In addition to osimeritinib, several third-generation agents have been investigated. In two phase I/II trials, lazertinib demonstrated an iORR of $44-85.7 \%$ in patients with $\mathrm{BrM}$ secondary to EGFR-mutant NSCLC $(130,131)$. Furmonertinib (formerly, alflutinib) exhibited good treatment efficacy in a phase I/II trial involving 17 EGFR-mutant NSCLC patients with BrM (iORR: 58.8\%; median PFS: 9.9 months) (132). These findings were replicated in a phase IIb trial involving 105 EGFR-mutant NSCLC patients with BrM: In 29 patients with one or more measurable BrM, treatment with alflutinib achieved an iORR of $66 \%$, while among the 87 patients in the complete analysis dataset, iORR was $34 \%$ and median PFS was 11.6 months
(95\% CI 8.3-13.8). Across both cohorts, the intracranial disease control rate ranged from $98-100 \%$ (133). Several clinical trials have also reported encouraging unpublished results, including a phase 2 expansion of the APOLLO trial (NCT02981108) on the efficacy of almonertinib in metastatic EGFR-mutant NSCLC. Further trials (NCT04808752, NCT04870190) are currently active to clarify the therapeutic efficacy of almonertinib. Thirdgeneration mutant EGFR inhibitors, such as rezivertinib and abivertinib (formerly, avitinib), have been investigated in patients with NSCLC. However, outcomes in subpopulations of patients with BrM have yet to be reported in published formats.

In addition to third-generation EGFR inhibitors, amivantamab, a bispecific antibody directed against EGFR and mesenchymal-epithelial transition (MET) receptor, has been investigated for the treatment of exon 20 insertion-EGFRmutant NSCLC. Initial phase I trial data from CHRYSALIS was encouraging: an ORR of $39 \%$ was achieved in patients with a history of IMD (134). A new clinical trial, MARIPOSA (NCT04487080), has recently been launched to compare the therapeutic efficacy of the combination of amivantamab and lazertinib with the efficacy of osimertinib in patients with untreated EGFR-mutant NSCLC.

\subsubsection{Emerging Targeted Therapies in NSCLC}

Mutations in the proto-oncogene KRAS have been reported in $25-30 \%$ of patients with NSCLC and, therefore, represent the most prevalent genomic driver of malignancy in the disease (160). As of December 2021, a single small molecule inhibitor of KRAS, sotorasib, has been developed and approved for the treatment of patients with KRAS ${ }^{\mathrm{G} 12 \mathrm{C}}$-positive NSCLC who have previously failed standard therapies; however, its clinical efficacy in the context of IMD remains to be determined (143). Selpercatinib and pralsetinib, inhibitors of the RET pathway, have shown promising systemic and CNS activity in RET fusionpositive disease $(144,145)$. Repotrectinib and lorlatinib have also been shown to have efficacy and CNS activity in patients with ROS1-positive NSCLC $(128,146)$. Tepotinib and capmatinib have demonstrated efficacy with good CNS activity in patients in NSCLC with MET exon 14 skipping mutations (161, 162). Finally, larotrectinib exhibited complete or partial intracranial responses in $2 / 3$ patients with BrM secondary to TRK fusionpositive cancers (163). Similar intracranial efficacy was demonstrated with entrectinib in patients with BrM secondary to TRK fusion-positive cancers (iORR 50-62.5\%) (164). The therapeutic efficacy of larotrectinib and entrectinib in TRK fusion-positive NSCLC specifically remains unclear, however, since these trials did not stratify patient outcomes by primary cancer type $(163,164)$.

\subsubsection{Targeted Therapies in Melanoma}

Studies have suggested that half of patients with metastatic melanoma will develop IMD, with a median OS of 4.7 months (165). Of note, genetic alterations in BRAF, including the activating mutations $\mathrm{BRAF}^{\mathrm{V} 600 \mathrm{E}}$ and $\mathrm{BRAF}^{\mathrm{V} 600 \mathrm{~K}}$, have been identified in $47 \%$ of patients with melanoma and in $24 \%$ of melanoma patients with IMD, making BRAF mutations a likely target in the treatment of IMD secondary to melanoma (166). 
In the landmark BREAK-MB trial, dabrafenib, a small molecule inhibitor of $\mathrm{BRAF}^{\mathrm{V} 600 \mathrm{E}}$, demonstrated adequate anti-tumor activity in $39.2 \%$ of $\mathrm{BRAF}^{\mathrm{V} 600 \mathrm{E}}$-positive melanoma patients with treatment-naïve IMD and $30.8 \%$ of $\mathrm{BRAF}^{\mathrm{V} 600 \mathrm{E}}$-positive melanoma patients with progressive IMD (147). Median OS was 33.1 weeks and 31.4 weeks in $\mathrm{BRAF}^{\mathrm{V} 600 \mathrm{E}}$-positive melanoma patients with treatment-naïv IMD and progressive IMD, respectively, and median PFS was 16.1 and 16.6 weeks in the same treatment groups. Intracranial response rates, median OS, and median PFS were lower for patients with $\mathrm{BRAF}^{\mathrm{V} 600 \mathrm{~K}}$ positive melanoma with progressive IMD than with treatmentnaïve IMD, though no explicit comparisons were made between cohorts (147). A second BRAF ${ }^{\mathrm{V} 600}$ inhibitor, vemurafenib, demonstrated adequate anti-tumor activity in $18 \%$ of patients with $\mathrm{BRAF}^{\mathrm{V} 600}$-positive melanoma with either untreated or progressive IMD (149). Median OS was 8.9 months in patients with untreated IMD and 9.6 months in patients with previously treated IMD, however, no comparisons were made with dabrafenib (149).

In the COMBI-MB trial, the combination of dabrafenib and the mitogen-activated protein kinase kinase (MEK) inhibitor trametinib demonstrated adequate intracranial tumor control in $58 \%$ of $\mathrm{BRAF}^{\mathrm{V} 600 \mathrm{E}}$-positive melanoma patients with treatmentnaïve, asymptomatic IMD, $56 \%$ of $\mathrm{BRAF}^{\mathrm{V} 600 \mathrm{E}}$-positive melanoma patients with progressive, asymptomatic IMD, $44 \%$ of BRAF $\mathrm{B}^{\mathrm{V} 600 \mathrm{D} / \mathrm{K} / \mathrm{R}}$-positive melanoma patients with asymptomatic IMD, and $59 \%$ of $\mathrm{BRAF}^{\mathrm{V} 600 \mathrm{E} / \mathrm{D} / \mathrm{K} / \mathrm{R}}$-positive melanoma with symptomatic IMD (148). Median OS was 10.8 and 24.3 months in $\mathrm{BRAF}^{\mathrm{V} 600 \mathrm{E}}$-positive melanoma patients with treatment-naïve IMD and progressive IMD, respectively. Median PFS was 5.6 and 7.2 months in the same cohorts (148). Several additional single or combination agents, including vemurafenib and the MEK inhibitor cobimetinib or the BRAF inhibitor encorafenib and MEK inhibitor binimetinib, have been described to be effective in treating IMD secondary to BRAFmutant melanoma. While these discussions have been thus far limited to case series and conference abstracts, early data suggest that multidrug targeted drug regimens for IMD secondary to BRAF-mutant melanoma may be future treatments for patients with BRAF-mutant melanoma and IMD.

\subsubsection{Immunotherapies}

Immune-checkpoint inhibitors (ICIs) include large monoclonal antibody-based therapies and small molecule inhibitors that upregulate the immune system and its antitumor activity (167, 168). Initial trials with ICIs have supported their use in patients with IMD secondary to NSCLC and melanoma. Additional trials are now underway to study their efficacy in patients with IMD secondary to other primary cancers, including breast cancer. For example, an early phase II trial studying ipilimumab, an anticytotoxic T-lymphocyte-associated antigen 4 (CLTA-4) antibody that promotes $\mathrm{T}$ lymphocyte destruction of cancer cells, demonstrated that $16 \%$ of melanoma patients with asymptomatic IMD receiving ipilimumab and 5\% of melanoma patients with symptomatic IMD receiving corticosteroids and ipilimumab achieved an intracranial objective response (169). In the same study, median OS was 7 months in melanoma patients with asymptomatic IMD receiving ipilimumab and 4 months in melanoma patients with symptomatic IMD receiving corticosteroids and ipilimumab.

In addition to CLTA-4, programmed cell death protein (PD1 ), which acts as a negative immune inhibitor, has emerged as a potential anti-cancer target. In an early phase II trial studying the anti-PD-1 antibody pembrolizumab, $22 \%$ of melanoma patients with IMD and $33 \%$ of NSCLC patients with IMD treated with pembrolizumab monotherapy demonstrated an intracranial objective response (170). A slightly lower iORR was found with the administration of the anti-PD-1 antibody nivolumab in patients with NSCLC and IMD (17\%) (171). Both studies support the hypothesis that anti-PD-1 antibodies are active in patients with IMD and present future therapy options. The combination of ipilimumab and nivolumab was investigated in Checkmate 204, which demonstrated an iORR in $53.5 \%$ of melanoma patients with asymptomatic IMD and $16.7 \%$ of melanoma patients with symptomatic IMD. In the same study, median PFS was reported to be 39.3 months and 1.2 months, respectively $(172,173)$. Finally, the RELATIVITY-047 trial recently reported prolonged PFS in patients with untreated or unresectable melanoma treated with a combination of nivolumab and lymphocyte-activation gene 3 (LAG-3) inhibitor relatlimab compared to those treated with nivolumab alone (HR 0.75, 95\% CI 0.62-0.92, p=0.006) (174). A subgroup analysis of patients with $\mathrm{BrM}$ and associated intracranial outcomes is necessary to clarify the role of this promising drug combination in the setting of IMD secondary to melanoma.

Monoclonal antibodies against programmed cell death-ligand 1 (PD-L1) have also been investigated. In patients with IMD secondary to TNBC studied in the Impassion130 RCT, the combination of atezolizumab and nab-paclitaxel did not significantly improve median PFS (HR 0.86, 95\% CI 0.50-1.49) or median OS (HR 1.34, 95\% CI 0.72-2.48) compared with placebo and nab-paclitaxel $(175,176)$. Additional prospective trials, including NCT03483012 and NCT04303988, may further clarify the role of PD-L1 inhibitors in patients with IMD and TNBC.

Further investigations are necessary to clarify the role of immunotherapy for IMD in patients receiving concurrent targeted therapies (Table 2).

\subsubsection{Immunotherapy in Combination With Radiation Therapy}

Results from studies investigating the combined administration of radiation and immunotherapies suggest a biological synergy between the two modalities $(182,183)$. Yet, evidence on the treatment of IMD with radiation and ICI is conflicting and limited to retrospective analyses. Knisley et al., for example, reported a median OS of 21.3 months in 27 patients with melanoma and BrM who received ipilimumab in combination with radiosurgery, compared with 4.9 months in 50 patients treated with radiosurgery alone $(\mathrm{p}=0.03)$ (178). These findings have been corroborated in another single-institution case series (median OS 18.3 vs. 5.3 months, HR 0.43, p=0.005) (179). On the other hand, investigators from New York University found no improvement in median OS when SRS was administered 
together with ipilimumab ( $\mathrm{n}=25)$ compared with SRS as a standalone therapy $(n=33)$ in patients with patients with melanoma and BrM (180). Several case reports also describe the combined administration of WBRT and immunotherapies, but evidence from larger cohort studies or RCTs is lacking $(184,185)$.

There is emerging evidence supporting the use of SRS and nivolumab: Minniti and colleagues reported that nivolumab was more effective in preventing intracranial disease progression and prolonging OS than ipilimumab when either agent was combined with SRS (median PFS 10 vs. 6 months, $\mathrm{p}=0.02$; median OS 22.0 vs. 14.7 months, $\mathrm{p}=0.015)$ (181). In a study of patients with renal cell carcinoma and IMD, the PFS benefits of nivolumab were enhanced by prior SRS or WBRT (median iPFS 2.7 vs. 4.8 months, HR 0.49, $\mathrm{p}=0.0277$ ) (177). Caution needs to be exercised around the current level of evidence, however. One 2013 RCT for example that compared WBRT plus SRS alone versus WBRT plus SRS in combination with temozolomide or erlotinib for NSCLC patients with limited number of metastases showed higher rates of grade 3 to 5 toxicities in the combination arms (11\%, 41\%, and 49\%, respectively, p<0.001) (186). Moreover, BrM may harbour a distinct set of genetic alterations compared with the primary lesion, and thus responses to targeted therapies may be limited (187). Given the success of immunotherapies in the treatment of IMD and the already-established utility of radiation therapy, these combined approaches are promising avenues for the future. Further research is required before these approaches can be reliably translated into clinical practice (Table 2).

\section{EMERGING CONSIDERATIONS FOR SECONDARY PREVENTION}

Given its impact on survival and QoL, there is clinical interest in early identification of IMD in patients with high-risk primary cancer types. Understanding the pathobiology of IMD may enable the development of new clinical tools to detect early IMD and initiate appropriate treatments in patients with lower systemic metastatic disease burden.

\subsection{Screening for IMD}

Experience from screening efforts for breast, prostate, and lung cancer have shown that early cancer detection and treatment results in improved disease control and prolonged survival (188-190). To date, IMD diagnosis has depended on imaging, either for staging or screening purposes, or to assess patients who manifest neurological symptoms concerning for brain metastases (7). Default intracranial imaging for all cancer patients would be structurally and financially infeasible; further, given the finding in observational studies that nearly $4 \%$ of asymptomatic individuals harbor an intracranial "incidentaloma", this approach would likely lead to overdiagnosis and unintentional overtreatment (191, 192). Instead, many current efforts have focused on screening of patients who are at high risk for the development of IMD (193). A recent review of studies reporting on the incidence of IMD in patients with metastatic and non-metastatic breast cancer found a significantly higher incidence of IMD in patients with metastatic HER2-positive (22-36\%) and metastatic TNBC (15-37\%) compared with other forms of metastatic breast cancer (approximately 10\%) and non-metastatic breast cancer (annual incidence of IMD as first site of recurrence $\leq 3 \%$ for all identified studies) (193). Findings from this review indicate that patients with metastatic HER2-positive and TNBC are at a sufficiently high risk for development of IMD to warrant routine screening. The American Society of Clinical Oncology (ASCO) and the NCCN currently do not recommend routine screening for IMD in women with metastatic breast cancer; conversely, the 2021 European Association of Neuro-Oncology (EANO)/European Society for Medical Oncology (ESMO) guidelines indicate that screening at diagnosis is "potentially justified" in patients with metastatic HER2-positive and TNBC [EANO: IV, n/a; ESMO: IV, B] (194). To clarify the risks and benefits of routine screening in these patients, multiple studies are currently randomizing patients with HER2-positive or TNBC, both subgroups with a well-defined higher risk of IMD, to either receive regularly scheduled MRI brain imaging or standard of care alone (NCT03881605; NCT04030507; NCT03617341) (193, 195). It remains to be determined if these efforts will result in improved outcomes for patients.

Overall, the reliance on imaging, and particularly MRI, has been a profound limitation to efforts for IMD screening, early detection, and treatment. There has been significant interest in development tools for IMD screening that circumvent intracranial imaging, for example, liquid biopsy (196). The concept of liquid biopsy rests on the assumption that different elements of tumor material, such as tumor-specific DNA, RNA, proteins, and exosomes, and circulating tumor cells, can be identified in blood or cerebrospinal fluid (CSF) as a surrogate for cancer burden. To date, these efforts have been limited by the need to isolate and enrich cancer markers in blood to enable sequencing for genomic approaches, which results in technical error and false biological signals, leading to high falsenegative results. There are also valid concerns that spillage of intracranial tumor into the systemic circulation may be limited, which could mandate study of CSF rather than blood (a much more invasive process which requires lumbar puncture) to assess intracranial disease (197).

\subsection{Understanding the Biology of Brain Metastases}

There has been significant interest in delineating the biological processes that underlie metastatic progression in cancer and, in particular, mediate the development of IMD (198). Multiple studies have shown that BrM are derived from cancer subclones that are distinct from dominant populations in the systemic cancer cell pool $(199,200)$. This finding raises the possibility that primary tumor profiling could identify patients who harbor subclones that are organotypic for the brain and are thus at risk of IMD (201). Further, this finding promises the likelihood of novel pathways, both cell-intrinsic and environmental, that are critical for IMD development and that could be targets for IMD prevention $(187,202,203)$. 
TABLE 2 | Summary of studies investigating immunotherapies for IMD.

\begin{tabular}{|c|c|c|c|c|c|c|c|c|}
\hline $\begin{array}{l}\text { Trial/ } \\
\text { Study }\end{array}$ & Drug & Radiation & Study design & $\begin{array}{c}\text { Total } \\
\text { participants } \\
\text { (n) }\end{array}$ & Cohorts & $\begin{array}{l}\text { Median OS } \\
\text { (months) }\end{array}$ & $\begin{array}{l}\text { Median PFS } \\
\text { (months) }\end{array}$ & Findings \\
\hline \multirow{4}{*}{$\begin{array}{l}\text { Margolin } \\
\text { et al. (169) }\end{array}$} & \multirow[t]{4}{*}{ Ipilimumab } & \multirow[t]{4}{*}{-} & \multirow{4}{*}{$\begin{array}{l}\text { Single-arm } \\
\text { clinical trial }\end{array}$} & \multirow[t]{4}{*}{72} & Asymptomatic IMD & 7 & - & iORR: 16\% \\
\hline & & & & & & & & Intracranial DCR: 24\% \\
\hline & & & & & \multirow{2}{*}{$\begin{array}{l}\text { Symptomatic IMD + } \\
\text { corticosteroids }\end{array}$} & \multirow[t]{2}{*}{4} & \multirow[t]{2}{*}{-} & iORR: $5 \%$ \\
\hline & & & & & & & & Intracranial DCR: 10\% \\
\hline \multirow{2}{*}{$\begin{array}{l}\text { Goldberg } \\
\text { et al. (170) }\end{array}$} & \multirow[t]{2}{*}{ Pembrolizumab } & \multirow[t]{2}{*}{-} & \multirow{2}{*}{$\begin{array}{l}\text { Single-arm } \\
\text { clinical trial }\end{array}$} & \multirow[t]{2}{*}{52} & Melanoma & NR & - & iORR: 22\% \\
\hline & & & & & NSCLC & 7.7 & - & iORR: 33\% \\
\hline \multirow{2}{*}{$\begin{array}{l}\text { Crinò et al. } \\
(171)\end{array}$} & \multirow[t]{2}{*}{ Nivolumab } & \multirow[t]{2}{*}{-} & \multirow{2}{*}{$\begin{array}{l}\text { Single-arm } \\
\text { clinical trial }\end{array}$} & \multirow[t]{2}{*}{1588} & \multirow[t]{2}{*}{-} & \multirow[t]{2}{*}{8.6} & \multirow[t]{2}{*}{3.0} & Overall ORR: 17\% \\
\hline & & & & & & & & Overall DCR: 39\% \\
\hline \multirow{4}{*}{$\begin{array}{l}\text { Flippot } \\
\text { et al. (177) }\end{array}$} & \multirow[t]{4}{*}{ Nivolumab } & \multirow[t]{4}{*}{-} & \multirow{4}{*}{$\begin{array}{l}\text { Single-arm } \\
\text { clinical trial }\end{array}$} & \multirow[t]{4}{*}{73} & Untreated IMD & \multirow[t]{2}{*}{-} & \multirow[t]{2}{*}{2.4} & iORR: $12 \%$ \\
\hline & & & & & & & & Intracranial DCR: 50\% \\
\hline & & & & & & & & Intracranial PFS: 2.7 months \\
\hline & & & & & $\begin{array}{l}\text { Previously treated } \\
\text { IMD (SRS/WBRT) }\end{array}$ & - & 2.5 & $\begin{array}{l}\text { Intracranial PFS: } 4.8 \\
\text { months, HR 0.49, } p=0.0277\end{array}$ \\
\hline Tawbi et al. & Ipilimumab + & - & Single-arm & 94 & - & - & - & iORR: $55 \%$ \\
\hline & nivolumab & & clinical trial & & & & & Intracranial DCR: 57\% \\
\hline Tawbi et al. & Ipilimumab + & - & Single-arm & 165 & Asymptomatic IMD & - & 39.3 & iORR: $53.5 \%$ \\
\hline (173) & nivolumab & & clinical trial & & & & & Intracranial DCR: 57.4\% \\
\hline & & & & & Symptomatic IMD & - & 1.2 & iORR: $16.7 \%$ \\
\hline & & & & & & & & Intracranial DCR: 16.7\% \\
\hline $\begin{array}{l}\text { Tawbi et al. } \\
(174)\end{array}$ & $\begin{array}{l}\text { Relatlimab + } \\
\text { nivolumab }\end{array}$ & - & $\mathrm{RCT}$ & 714 & $\begin{array}{l}\text { Relatlimab + } \\
\text { nivolumab }\end{array}$ & - & - & - \\
\hline & & & & & Nivolumab & - & - & - \\
\hline $\begin{array}{l}\text { Schmid } \\
\text { et al. (175) }\end{array}$ & $\begin{array}{l}\text { Atezolizumab + } \\
\text { nab-paclitaxel }\end{array}$ & - & $\mathrm{RCT}$ & 451 & $\begin{array}{l}\text { Atezolizumab + nab- } \\
\text { paclitaxel }\end{array}$ & - & $\begin{array}{c}4.9 \\
(\mathrm{HR} 0.86,95 \% \\
\mathrm{Cl} 0.50-1.49)\end{array}$ & - \\
\hline & & & & & $\begin{array}{l}\text { Placebo + nab- } \\
\text { paclitaxel }\end{array}$ & - & 4.4 & - \\
\hline $\begin{array}{l}\text { Schmid } \\
\text { et al. (176) }\end{array}$ & $\begin{array}{l}\text { Atezolizumab + } \\
\text { nab-paclitaxel }\end{array}$ & - & $\mathrm{RCT}$ & 902 & $\begin{array}{l}\text { Atezolizumab + nab- } \\
\text { paclitaxel }\end{array}$ & $\begin{array}{c}14.3 \\
(\mathrm{HR} 1.34,95 \% \\
\mathrm{Cl} 0.72-2.48)\end{array}$ & - & - \\
\hline & & & & & $\begin{array}{l}\text { Placebo + nab- } \\
\text { paclitaxel }\end{array}$ & 16.2 & - & - \\
\hline Knisely & Ipilimumab & SRS & Retrospective & 77 & SRS + ipilimumab & 21.3 & - & - \\
\hline et al. (178) & & & cohort study & & SRS only & $\begin{array}{c}4.9(\mathrm{HR} 0.48 \\
\quad p=0.03)\end{array}$ & - & - \\
\hline Silk et al. & Ipilimumab & SRS & Retrospective & 70 & SRS + ipilimumab & 18.3 & 2.7 & - \\
\hline (179) & & & cohort study & & SRS only & $\begin{array}{c}5.3(H R \text { 0.43 } \\
p=0.005)\end{array}$ & 3.3 & - \\
\hline $\begin{array}{l}\text { Mathew } \\
\text { et al. (180) }\end{array}$ & Ipilimumab & SRS & $\begin{array}{l}\text { Retrospective } \\
\text { cohort study }\end{array}$ & 58 & SRS + ipilimumab & $\begin{array}{l}56 \% \text { in } 6 \\
\text { months }\end{array}$ & - & - \\
\hline & & & & & SRS only & $\begin{array}{c}45 \% \text { in } 6 \\
\text { months }(p=0.18)\end{array}$ & - & - \\
\hline Minniti et al. & Nivolumab or & SRS & Retrospective & 80 & Nivolumab + SRS & 22.0 & 10 & iORR: 76\% \\
\hline$(181)$ & ipilimumab & & cohort study & & Ipilimumab + SRS & $14.7(p=0.015)$ & $6(p=0.02)$ & \\
\hline
\end{tabular}

Median overall survival marked with a dash if the data was 1) not reported or 2) reported for the entire population, including patients without IMD.

CI, confidence interval; DCR, disease control rate; HR, hazard ratio; IMD, intracranial metastatic disease; iORR, intracranial ORR; NR, not yet reached; NSCLC, non-small cell lung cancer; ORR, objective response rate; OS, overall survival; PFS, progression-free survival; RCT, randomized control trial; SRS, stereotactic radiosurgery; WBRT, whole-brain radiotherapy.

\section{CONCLUSION}

Diagnosis of IMD places a significant burden on patient survival and QoL. Over the past several decades, technical innovations and an advancing understanding of tumor biology have enabled physicians to optimize treatment outcomes for these patients. While WBRT initially formed the mainstay of localized treatment for IMD, surgical resection and SRS have become established treatment approaches for patients with limited intracranial disease burden and are increasingly considered for a wider patient spectrum. Several molecular drivers have also been identified as targets for systemic therapy. While most of these agents historically had limited application for treatment of IMD, mounting evidence suggests that some targeted therapy drugs may retain activity in the brain, especially in patients with IMD due to single driver-mediated breast cancer, NSCLC, and melanoma. Early investigations into the efficacy of immunotherapies and their combination with radiation therapy may further form future avenues of treatment. These advances promise to improve outcomes patients with cancer and IMD. 


\section{AUTHOR CONTRIBUTIONS}

$\mathrm{AL}$ and $\mathrm{KG}$ contributed equally to the design and writing of the manuscript and share first authorship. SD supervised this work. All authors contributed to the article and approved the submitted version.

\section{REFERENCES}

1. Scoccianti S, Ricardi U. Treatment of Brain Metastases: Review of Phase III Randomized Controlled Trials. Radiother Oncol (2012) 102(2):168-79. doi: 10.1016/j.radonc.2011.08.041

2. Habbous S, Forster K, Darling G, Jerzak K, Holloway CMB, Sahgal A, et al. Incidence and Real-World Burden of Brain Metastases From Solid Tumors and Hematologic Malignancies in Ontario: A Population-Based Study. Neurooncol Adv (2021) 3(1):vdaa178. doi: 10.1093/noajnl/vdaa178

3. Zakaria R, Das K, Bhojak M, Radon M, Walker C, Jenkinson MD. The Role of Magnetic Resonance Imaging in the Management of Brain Metastases: Diagnosis to Prognosis. Cancer Imaging (2014) 14(1):8. doi: 10.1186/14707330-14-8

4. Winther-Larsen A, Hviid CVB, Meldgaard P, Sorensen BS, SandfeldPaulsen B. Neurofilament Light Chain as A Biomarker for Brain Metastases. Cancers (2020) 12(10):2852. doi: 10.3390/cancers 12102852

5. Bhatia A, Birger M, Veeraraghavan H, Um H, Tixier F, McKenney AS, et al. MRI Radiomic Features Are Associated With Survival in Melanoma Brain Metastases Treated With Immune Checkpoint Inhibitors. Neuro-Oncology (2019) 21(12):1578-86. doi: 10.1093/neuonc/noz141

6. Sperduto PW, Mesko S, Li J, Cagney D, Aizer A, Lin NU, et al. Survival in Patients With Brain Metastases: Summary Report on the Updated Diagnosis-Specific Graded Prognostic Assessment and Definition of the Eligibility Quotient. J Clin Oncol (2020) 38(32):3773-84. doi: 10.1200/ JCO.20.01255

7. Suh JH, Kotecha R, Chao ST, Ahluwalia MS, Sahgal A, Chang EL. Current Approaches to the Management of Brain Metastases. Nat Rev Clin Oncol (2020) 17(5):279-99. doi: 10.1038/s41571-019-0320-3

8. Borgelt B, Gelber R, Kramer S, Brady LW, Chang CH, Davis LW, et al. The Palliation of Brain Metastases: Final Results of the First Two Studies by the Radiation Therapy Oncology Group. Int J Radiat Oncol Biol Phys (1980) 6 (1):1-9. doi: 10.1016/0360-3016(80)90195-9

9. Mehta MP, Rodrigus P, Terhaard CH, Rao A, Suh J, Roa W, et al. Survival and Neurologic Outcomes in a Randomized Trial of Motexafin Gadolinium and Whole-Brain Radiation Therapy in Brain Metastases. J Clin Oncol (2003) 21(13):2529-36. doi: 10.1200/JCO.2003.12.122

10. Suh JH, Stea B, Nabid A, Kresl JJ, Fortin A, Mercier JP, et al. Phase III Study of Efaproxiral as an Adjunct to Whole-Brain Radiation Therapy for Brain Metastases. J Clin Oncol (2006) 24(1):106-14. doi: 10.1200/ JCO.2004.00.1768

11. Sperduto PW, Deegan BJ, Li J, Jethwa KR, Brown PD, Lockney N, et al. Estimating Survival for Renal Cell Carcinoma Patients With Brain Metastases: An Update of the Renal Graded Prognostic Assessment Tool. Neuro Oncol (2018) 20(12):1652-60. doi: 10.1093/neuonc/noy099

12. Niranjan A, Lunsford LD, Ahluwalia MS. Targeted Therapies for Brain Metastases. Prog Neurol Surg (2019) 34:125-37. doi: 10.1159/000493057

13. Erickson AW, Habbous S, Wright F, Lofters AK, Jerzak KJ, Das S. Assessing the Association of Targeted Therapy and Intracranial Metastatic Disease. JAMA Oncol (2021) 7(8):1220-4. doi: 10.1001/jamaoncol.2021.1600

14. Kanner AA, Bokstein F, Blumenthal DT, Ram Z. Surgical Therapies in Brain Metastasis. Semin Oncol (2007) 34(3):197-205. doi: 10.1053/ j.seminoncol.2007.03.011

15. Kuo T, Recht L. Optimizing Therapy for Patients With Brain Metastases. Semin Oncol (2006) 33(3):299-306. doi: 10.1053/j.seminoncol.2006.03.005

16. Sawaya R, Hammoud M, Schoppa D, Hess KR, Wu SZ, Shi WM, et al. Neurosurgical Outcomes in a Modern Series of 400 Craniotomies for Treatment of Parenchymal Tumors. Neurosurgery (1998) 42(5):1044-55; discussion 55-6. doi: 10.1097/00006123-199805000-00054

\section{FUNDING}

KG is supported by the Graduate Diploma in Health Research at the University of Toronto. SD is supported by the Canadian Institute for Health Research and Gratitude 10. The funding source had no role in the preparation and submission of this review article.

17. Redmond KJ, De Salles AAF, Fariselli L, Levivier M, Ma L, Paddick I, et al Stereotactic Radiosurgery for Postoperative Metastatic Surgical Cavities: A Critical Review and International Stereotactic Radiosurgery Society (ISRS) Practice Guidelines. Int J Radiat Oncol Biol Phys (2021) 111(1):68-80. doi: 10.1016/j.ijrobp.2021.04.016

18. Prabhu RS, Turner BE, Asher AL, Marcrom SR, Fiveash JB, Foreman PM, et al. A Multi-Institutional Analysis of Presentation and Outcomes for Leptomeningeal Disease Recurrence After Surgical Resection and Radiosurgery for Brain Metastases. Neuro Oncol (2019) 21(8):1049-59. doi: 10.1093/neuonc/noz049

19. DeAngelis LM, Posner JB. Neurologic Complications of Cancer. New York City, NY, USA: Oxford University Press (2009).

20. Patchell RA, Tibbs PA, Walsh JW, Dempsey RJ, Maruyama Y, Kryscio RJ, et al. A Randomized Trial of Surgery in the Treatment of Single Metastases to the Brain. N Engl J Med (1990) 322(8):494-500. doi: 10.1056/ NEJM199002223220802

21. Vecht CJ, Haaxma-Reiche H, Noordijk EM, Padberg GW, Voormolen JH, Hoekstra FH, et al. Treatment of Single Brain Metastasis: Radiotherapy Alone or Combined With Neurosurgery? Ann Neurol (1993) 33(6):583-90. doi: 10.1002/ana.410330605

22. Mintz AH, Kestle J, Rathbone MP, Gaspar L, Hugenholtz H, Fisher B, et al. A Randomized Trial to Assess the Efficacy of Surgery in Addition to Radiotherapy in Patients With a Single Cerebral Metastasis. Cancer (1996) 78(7):1470-6. doi: 10.1002/(SICI)1097-0142(19961001)78:7<1470::AID CNCR14>3.0.CO;2-X

23. Gaspar LE, Mehta MP, Patchell RA, Burri SH, Robinson PD, Morris RE, et al. The Role of Whole Brain Radiation Therapy in the Management of Newly Diagnosed Brain Metastases: A Systematic Review and EvidenceBased Clinical Practice Guideline. J Neurooncol (2010) 96(1):17-32. doi: 10.1007/s11060-009-0060-9

24. Kalkanis SN, Kondziolka D, Gaspar LE, Burri SH, Asher AL, Cobbs CS, et al. The Role of Surgical Resection in the Management of Newly Diagnosed Brain Metastases: A Systematic Review and Evidence-Based Clinical Practice Guideline. J Neurooncol (2010) 96(1):33-43. doi: 10.1007/s11060-009-0061-8

25. Garell PC, Hitchon PW, Wen BC, Mellenberg DE, Torner J. Stereotactic Radiosurgery Versus Microsurgical Resection for the Initial Treatment of Metastatic Cancer to the Brain. J Radiosurg (1999) 2(1):1-5. doi: 10.1023/ A:1022914932190

26. O’Neill BP, Iturria NJ, Link MJ, Pollock BE, Ballman KV, O'Fallon JR. A Comparison of Surgical Resection and Stereotactic Radiosurgery in the Treatment of Solitary Brain Metastases. Int J Radiat Oncol Biol Phys (2003) 55(5):1169-76. doi: 10.1016/S0360-3016(02)04379-1

27. Schödel P, Schebesch K-M, Brawanski A, Proescholdt MA. Surgical Resection of Brain Metastases-Impact on Neurological Outcome. Int J Mol Sci (2013) 14(5):8708-18. doi: 10.3390/ijms14058708

28. Kondziolka D, Kano H, Harrison GL, Yang HC, Liew DN, Niranjan A, et al. Stereotactic Radiosurgery as Primary and Salvage Treatment for Brain Metastases From Breast Cancer. Clin article J Neurosurg (2011) 114 (3):792-800. doi: 10.3171/2010.8.JNS10461

29. Gazzeri R, Nalavenkata S, Teo C. Minimally Invasive Key-Hole Approach for the Surgical Treatment of Single and Multiple Brain Metastases. Clin Neurol Neurosurg (2014) 123:117-26. doi: 10.1016/j.clineuro.2014.05.010

30. Iwadate Y, Namba H, Yamaura A. Significance of Surgical Resection for the Treatment of Multiple Brain Metastases. Anticancer Res (2000) 20(1b):573-7.

31. Salvati M, Tropeano MP, Maiola V, Lavalle L, Brogna C, Colonnese C, et al. Multiple Brain Metastases: A Surgical Series and Neurosurgical Perspective. Neurol Sci (2018) 39(4):671-7. doi: 10.1007/s10072-017-3220-2

32. Baker CM, Glenn CA, Briggs RG, Burks JD, Smitherman AD, Conner AK, et al. Simultaneous Resection of Multiple Metastatic Brain Tumors With 
Multiple Keyhole Craniotomies. World Neurosurg (2017) 106:359-67. doi: 10.1016/j.wneu.2017.06.118

33. Eroglu U, Shah K, Bozkurt M, Kahilogullari G, Yakar F, Dogan İ, et al. Supraorbital Keyhole Approach: Lessons Learned From 106 Operative Cases. World Neurosurg (2019) 124:e667-74. doi: 10.1016/ j.wneu.2018.12.188

34. Gazzeri R, Nishiyama Y, Teo C. Endoscopic Supraorbital Eyebrow Approach for the Surgical Treatment of Extraaxialand Intraaxial Tumors. Neurosurg Focus (2014) 37(4):E20. doi: 10.3171/2014.7.FOCUS14203

35. Phang I, Leach J, Leggate JRS, Karabatsou K, Coope D, D'Urso PI. Minimally Invasive Resection of Brain Metastases. World Neurosurg (2019) 130:e362e7. doi: 10.1016/j.wneu.2019.06.091

36. Raza SM, Garzon-Muvdi T, Boaehene K, Olivi A, Gallia G, Lim M, et al. The Supraorbital Craniotomy for Access to the Skull Base and Intraaxial Lesions: A Technique in Evolution. Minim Invasive Neurosurg (2010) 53(1):1-8. doi: 10.1055/s-0030-1247504

37. Wang JL, Elder JB. Techniques for Open Surgical Resection of Brain Metastases. Neurosurg Clin N Am (2020) 31(4):527-36. doi: 10.1016/ j.nec.2020.06.003

38. Gassie K, Alvarado-Estrada K, Bechtle P, Chaichana KL. Surgical Management of Deep-Seated Metastatic Brain Tumors Using Minimally Invasive Approaches. J Neurol Surg A Cent Eur Neurosurg (2019) 80(3):198204. doi: $10.1055 / \mathrm{s}-0038-1676575$

39. Chao JH, Phillips R, Nickson JJ. Roentgen-Ray Therapy of Cerebral Metastases. Cancer (1954) 7(4):682-9. doi: 10.1002/1097-0142(195407) 7:4<682::AID-CNCR2820070409>3.0.CO;2-S

40. Order SE, Hellmän S, Essen CFV, Kligerman MM. Improvement in Quality of Survival Following Whole-Brain Irradiation for Brain Metastasis. Radiology (1968) 91(1):149-53. doi: 10.1148/91.1.149

41. Patchell RA, Tibbs PA, Regine WF, Dempsey RJ, Mohiuddin M, Kryscio RJ, et al. Postoperative Radiotherapy in the Treatment of Single Metastases to the Brain: A Randomized Trial. Jama (1998) 280(17):1485-9. doi: 10.1001/ jama.280.17.1485

42. Tallet AV, Azria D, Barlesi F, Spano JP, Carpentier AF, Gonçalves A, et al. Neurocognitive Function Impairment After Whole Brain Radiotherapy for Brain Metastases: Actual Assessment. Radiat Oncol (2012) 7:77. doi: 10.1186/1748-717X-7-77

43. Brown PD, Pugh S, Laack NN, Wefel JS, Khuntia D, Meyers C, et al. Memantine for the Prevention of Cognitive Dysfunction in Patients Receiving Whole-Brain Radiotherapy: A Randomized, Double-Blind, Placebo-Controlled Trial. NeuroOncology (2013) 15(10):1429-37. doi: 10.1093/neuonc/not114

44. Gondi V, Deshmukh S, Brown PD, Wefel JS, Tome W, Armstrong T, et al. NRG Oncology CC001: A Phase III Trial of Hippocampal Avoidance (HA) in Addition to Whole-Brain Radiotherapy (WBRT) Plus Memantine to Preserve Neurocognitive Function (NCF) in Patients With Brain Metastases (BM). Am Soc Clin Oncol (2019) 37(15 suppl):2009. doi: 10.1200/ JCO.2019.37.15_suppl.2009

45. Gondi V, Pugh SL, Tome WA, Caine C, Corn B, Kanner A, et al. Preservation of Memory With Conformal Avoidance of the Hippocampal Neural Stem-Cell Compartment During Whole-Brain Radiotherapy for Brain Metastases (RTOG 0933): A Phase II Multi-Institutional Trial. J Clin Oncol (2014) 32(34):3810-6. doi: 10.1200/JCO.2014.57.2909

46. Meyers CA, Smith JA, Bezjak A, Mehta MP, Liebmann J, Illidge T, et al. Neurocognitive Function and Progression in Patients With Brain Metastases Treated With Whole-Brain Radiation and Motexafin Gadolinium: Results of a Randomized Phase III Trial. J Clin Oncol (2004) 22(1):157-65. doi: 10.1200/ JCO.2004.05.128

47. Soffietti R, Abacioglu U, Baumert B, Combs SE, Kinhult S, Kros JM, et al. Diagnosis and Treatment of Brain Metastases From Solid Tumors: Guidelines From the European Association of Neuro-Oncology (EANO). Neuro Oncol (2017) 19(2):162-74. doi: 10.1093/neuonc/now241

48. Gerosa M, Nicolato A, Foroni R, Tomazzoli L, Bricolo A. Analysis of Long-Term Outcomes and Prognostic Factors in Patients With Non-Small Cell Lung Cancer Brain Metastases Treated by Gamma Knife Radiosurgery. J Neurosurg (2005) 102 Suppl:75-80. doi: 10.3171/jns.2005.102.s_supplement.0075

49. Hussain A, Brown PD, Stafford SL, Pollock BE. Stereotactic Radiosurgery for Brainstem Metastases: Survival, Tumor Control, and Patient Outcomes. Int J Radiat Oncol Biol Phys (2007) 67(2):521-4. doi: 10.1016/j.ijrobp.2006.08.081
50. Churilla TM, Chowdhury IH, Handorf E, Collette L, Collette S, Dong Y, et al. Comparison of Local Control of Brain Metastases With Stereotactic Radiosurgery vs Surgical Resection: A Secondary Analysis of a Randomized Clinical Trial. JAMA Oncol (2019) 5(2):243-7. doi: 10.1001/ jamaoncol.2018.4610

51. Andrews DW, Scott CB, Sperduto PW, Flanders AE, Gaspar LE, Schell MC, et al. Whole Brain Radiation Therapy With or Without Stereotactic Radiosurgery Boost for Patients With One to Three Brain Metastases: Phase III Results of the RTOG 9508 Randomised Trial. Lancet (2004) 363 (9422):1665-72. doi: 10.1016/S0140-6736(04)16250-8

52. Kondziolka D, Patel A, Lunsford LD, Kassam A, Flickinger JC. Stereotactic Radiosurgery Plus Whole Brain Radiotherapy Versus Radiotherapy Alone for Patients With Multiple Brain Metastases. Int J Radiat Oncol Biol Phys (1999) 45(2):427-34. doi: 10.1016/S0360-3016(99)00198-4

53. Aoyama H, Shirato H, Tago M, Nakagawa K, Toyoda T, Hatano K, et al. Stereotactic Radiosurgery Plus Whole-Brain Radiation Therapy vs Stereotactic Radiosurgery Alone for Treatment of Brain Metastases: A Randomized Controlled Trial. Jama (2006) 295(21):2483-91. doi: 10.1001/ jama.295.21.2483

54. Chang EL, Wefel JS, Hess KR, Allen PK, Lang FF, Kornguth DG, et al. Neurocognition in Patients With Brain Metastases Treated With Radiosurgery or Radiosurgery Plus Whole-Brain Irradiation: A Randomised Controlled Trial. Lancet Oncol (2009) 10(11):1037-44. doi: 10.1016/S1470-2045(09)70263-3

55. Weiss SE, Kelly PJ. Neurocognitive Function After WBRT Plus SRS or SRS Alone. Lancet Oncol (2010) 11(3):220-1. doi: 10.1016/S1470-2045(09) 70387-0

56. Kocher M, Soffietti R, Abacioglu U, Villà S, Fauchon F, Baumert BG, et al. Adjuvant Whole-Brain Radiotherapy Versus Observation After Radiosurgery or Surgical Resection of One to Three Cerebral Metastases: Results of the EORTC 22952-26001 Study. J Clin Oncol (2011) 29(2):134-41. doi: $10.1200 /$ JCO.2010.30.1655

57. Soffietti R, Kocher M, Abacioglu UM, Villa S, Fauchon F, Baumert BG, et al. A European Organisation for Research and Treatment of Cancer Phase III Trial of Adjuvant Whole-Brain Radiotherapy Versus Observation in Patients With One to Three Brain Metastases From Solid Tumors After Surgical Resection or Radiosurgery: Quality-of-Life Results. J Clin Oncol (2013) 31 (1):65-72. doi: 10.1200/JCO.2011.41.0639

58. Kim H, Rajagopalan MS, Beriwal S, Smith KJ. Cost-Effectiveness Analysis of Stereotactic Radiosurgery Alone Versus Stereotactic Radiosurgery With Upfront Whole Brain Radiation Therapy for Brain Metastases. Clin Oncol (R Coll Radiol) (2017) 29(10):e157-e64. doi: 10.1016/j.clon.2017.05.001

59. Sahgal A, Aoyama H, Kocher M, Neupane B, Collette S, Tago M, et al. Phase 3 Trials of Stereotactic Radiosurgery With or Without Whole-Brain Radiation Therapy for 1 to 4 Brain Metastases: Individual Patient Data Meta-Analysis. Int J Radiat Oncol Biol Phys (2015) 91(4):710-7. doi: 10.1016/j.ijrobp.2014.10.024

60. Tsao M, Xu W, Sahgal A. A Meta-Analysis Evaluating Stereotactic Radiosurgery, Whole-Brain Radiotherapy, or Both for Patients Presenting With a Limited Number of Brain Metastases. Cancer (2012) 118(9):2486-93. doi: $10.1002 / \mathrm{cncr} .26515$

61. Hong AM, Fogarty GB, Dolven-Jacobsen K, Burmeister BH, Lo SN, Haydu LE, et al. Adjuvant Whole-Brain Radiation Therapy Compared With Observation After Local Treatment of Melanoma Brain Metastases: A Multicenter, Randomized Phase III Trial. J Clin Oncol (2019) 37 (33):3132-41. doi: 10.1200/JCO.19.01414

62. ASTRO. Don't Routinely Add Adjuvant Whole Brain Radiation Therapy to Stereotactic Radiosurgery for Limited Brain Metastases. Philadelphia: ABIM Foundation (2014). Available at: https://www.choosingwisely.org/clinician-lists/ american-society-radiation-oncology-adjuvant-whole-brain-radiation-therapy/.

63. Yamamoto M, Serizawa T, Shuto T, Akabane A, Higuchi Y, Kawagishi J, et al. Stereotactic Radiosurgery for Patients With Multiple Brain Metastases (JLGK0901): A Multi-Institutional Prospective Observational Study. Lancet Oncol (2014) 15(4):387-95. doi: 10.1016/S1470-2045(14)70221-9

64. Blonigen BJ, Steinmetz RD, Levin L, Lamba MA, Warnick RE, Breneman JC. Irradiated Volume as a Predictor of Brain Radionecrosis After Linear Accelerator Stereotactic Radiosurgery. Int J Radiat Oncol Biol Phys (2010) 77(4):996-1001. doi: 10.1016/j.ijrobp.2009.06.006 
65. Chin LS, Ma L, DiBiase S. Radiation Necrosis Following Gamma Knife Surgery: A Case-Controlled Comparison of Treatment Parameters and Long-Term Clinical Follow Up. J Neurosurg (2001) 94(6):899-904. doi: 10.3171/jns.2001.94.6.0899

66. Kohutek ZA, Yamada Y, Chan TA, Brennan CW, Tabar V, Gutin PH, et al. Long-Term Risk of Radionecrosis and Imaging Changes After Stereotactic Radiosurgery for Brain Metastases. J Neuro-Oncol (2015) 125(1):149-56. doi: 10.1007/s11060-015-1881-3

67. Minniti G, Clarke E, Lanzetta G, Osti MF, Trasimeni G, Bozzao A, et al. Stereotactic Radiosurgery for Brain Metastases: Analysis of Outcome and Risk of Brain Radionecrosis. Radiat Oncol (2011) 6(1):1-9. doi: 10.1186/ 1748-717X-6-48

68. Varlotto JM, Flickinger JC, Niranjan A, Bhatnagar AK, Kondziolka D, Lunsford LD. Analysis of Tumor Control and Toxicity in Patients Who Have Survived at Least One Year After Radiosurgery for Brain Metastases. Int J Radiat Oncol Biol Phys (2003) 57(2):452-64. doi: 10.1016/S0360-3016 (03)00568-6

69. Fujimoto D, von Eyben R, Gibbs IC, Chang SD, Li G, Harsh GR, et al. Imaging Changes Over 18 Months Following Stereotactic Radiosurgery for Brain Metastases: Both Late Radiation Necrosis and Tumor Progression can Occur. J Neuro-Oncol (2018) 136(1):207-12. doi: 10.1007/s11060-0172647-x

70. Strenger V, Lackner H, Mayer R, Sminia P, Sovinz P, Mokry M, et al. Incidence and Clinical Course of Radionecrosis in Children With Brain Tumors. Strahlenther und Onkologie (2013) 189(9):759-64. doi: 10.1007/ s00066-013-0408-0

71. Chi D, Béhin A, Delattre J-Y. Neurologic Complications of Radiation Therapy. In: Cancer Neurology in Clinical Practice. Totowa, NJ: Humana Press (2008). p. 259-86.

72. Rahmathulla G, Marko NF, Weil RJ. Cerebral Radiation Necrosis: A Review of the Pathobiology, Diagnosis and Management Considerations. J Clin Neurosci (2013) 20(4):485-502. doi: 10.1016/j.jocn.2012.09.011

73. Ruben JD, Dally M, Bailey M, Smith R, McLean CA, Fedele P. Cerebral Radiation Necrosis: Incidence, Outcomes, and Risk Factors With Emphasis on Radiation Parameters and Chemotherapy. Int J Radiat Oncol Biol Phys (2006) 65(2):499-508. doi: 10.1016/j.ijrobp.2005.12.002

74. Nedzi LA, Kooy H, Alexander E, Gelman RS, Loeffler JS. Variables Associated With the Development of Complications From Radiosurgery of Intracranial Tumors. Int J Radiat OncolBiolPhys (1991) 21(3):591-9. doi: 10.1016/0360-3016(91)90675-T

75. Colaco RJ, Martin P, Kluger HM, Yu JB, Chiang VL. Does Immunotherapy Increase the Rate of Radiation Necrosis After Radiosurgical Treatment of Brain Metastases? J Neurosurg (2016) 125(1):17-23. doi: 10.3171/ 2015.6.JNS142763

76. Kim JM, Miller JA, Kotecha R, Xiao R, Juloori A, Ward MC, et al. The Risk of Radiation Necrosis Following Stereotactic Radiosurgery With Concurrent Systemic Therapies. J Neurooncol (2017) 133(2):357-68. doi: 10.1007/ s11060-017-2442-8

77. Sneed PK, Mendez J, Vemer-van den Hoek JG, Seymour ZA, Ma L, Molinaro AM, et al. Adverse Radiation Effect After Stereotactic Radiosurgery for Brain Metastases: Incidence, Time Course, and Risk Factors. J Neurosurg (2015) 123(2):373-86. doi: 10.3171/2014.10.JNS141610

78. Martin AM, Cagney DN, Catalano PJ, Alexander BM, Redig AJ, Schoenfeld JD, et al. Immunotherapy and Symptomatic Radiation Necrosis in Patients With Brain Metastases Treated With Stereotactic Radiation. JAMA Oncol (2018) 4(8):1123-4. doi: 10.1001/jamaoncol.2017.3993

79. Levin VA, Bidaut L, Hou P, Kumar AJ, Wefel JS, Bekele BN, et al. Randomized Double-Blind Placebo-Controlled Trial of Bevacizumab Therapy for Radiation Necrosis of the Central Nervous System. Int $J$ Radiat Oncol Biol Phys (2011) 79(5):1487-95. doi: 10.1016/ j.ijrobp.2009.12.061

80. McPherson CM, Warnick RE. Results of Contemporary Surgical Management of Radiation Necrosis Using Frameless Stereotaxis and Intraoperative Magnetic Resonance Imaging. J Neurooncol (2004) 68 (1):41-7. doi: 10.1023/B:NEON.0000024744.16031.e9

81. Newman WC, Goldberg J, Guadix SW, Brown S, Reiner AS, Panageas K, et al. The Effect of Surgery on Radiation Necrosis in Irradiated Brain Metastases: Extent of Resection and Long-Term Clinical and Radiographic
Outcomes. J Neurooncol (2021) 153(3):507-18. doi: 10.1007/s11060-02103790-y

82. Ahluwalia M, Barnett GH, Deng D, Tatter SB, Laxton AW, Mohammadi AM, et al. Laser Ablation After Stereotactic Radiosurgery: A Multicenter Prospective Study in Patients With Metastatic Brain Tumors and Radiation Necrosis. J Neurosurg (2018) 130(3):804-11. doi: 10.3171/ 2017.11.JNS171273

83. Lamba N, Mehanna E, Kearney RB, Catalano PJ, Brown PD, Haas-Kogan DA, et al. Prescription of Memantine During Non-Stereotactic, BrainDirected Radiation Among Patients With Brain Metastases: A PopulationBased Study. J Neuro-Oncol (2020) 148(3):509-17. doi: 10.1007/s11060-02003542-4

84. Attia A, Rapp SR, Case LD, D’Agostino R, Lesser G, Naughton M, et al. Phase II Study of Ginkgo Biloba in Irradiated Brain Tumor Patients: Effect on Cognitive Function, Quality of Life, and Mood. J Neurooncol (2012) 109 (2):357-63. doi: 10.1007/s11060-012-0901-9

85. Rapp SR, Case LD, Peiffer A, Naughton MM, Chan MD, Stieber VW, et al. Donepezil for Irradiated Brain Tumor Survivors: A Phase III Randomized Placebo-Controlled Clinical Trial. J Clin Oncol (2015) 33(15):1653-9. doi: 10.1200/JCO.2014.58.4508

86. Shaw MG, Ball DL. Treatment of Brain Metastases in Lung Cancer: Strategies to Avoid/Reduce Late Complications of Whole Brain Radiation Therapy. Curr Treat Options Oncol (2013) 14(4):553-67. doi: 10.1007/ s11864-013-0258-0

87. Robbins ME, Payne V, Tommasi E, Diz DI, Hsu FC, Brown WR, et al. The AT1 Receptor Antagonist, L-158,809, Prevents or Ameliorates Fractionated Whole-Brain Irradiation-Induced Cognitive Impairment. Int J Radiat Oncol Biol Phys (2009) 73(2):499-505. doi: 10.1016/j.ijrobp.2008.09.058

88. Monje ML, Mizumatsu S, Fike JR, Palmer TD. Irradiation Induces Neural Precursor-Cell Dysfunction. Nat Med (2002) 8(9):955-62. doi: 10.1038/ nm749

89. Ghia A, Tomé WA, Thomas S, Cannon G, Khuntia D, Kuo JS, et al. Distribution of Brain Metastases in Relation to the Hippocampus: Implications for Neurocognitive Functional Preservation. Int J Radiat Oncol Biol Phys (2007) 68(4):971-7. doi: 10.1016/j.ijrobp.2007.02.016

90. Brown PD, Gondi V, Pugh S, Tome WA, Wefel JS, Armstrong TS, et al. Hippocampal Avoidance During Whole-Brain Radiotherapy Plus Memantine for Patients With Brain Metastases: Phase III Trial NRG Oncology Cc001. J Clin Oncol (2020) 38(10):1019-29. doi: 10.1200/ JCO.19.02767

91. Rusthoven CG, Camidge DR, Robin TP, Brown PD. Radiosurgery for SmallCell Brain Metastases: Challenging the Last Bastion of Preferential WholeBrain Radiotherapy Delivery. J Clin Oncol (2020) 38(31):3587-91. doi: 10.1200/JCO.20.01823

92. Higgins KA, Simone CB2nd, Amini A, Chetty IJ, Donington J, Edelman MJ, et al. American Radium Society Appropriate Use Criteria on Radiation Therapy for Extensive-Stage SCLC. J Thorac Oncol (2021) 16(1):54-65. doi: 10.1016/j.jtho.2020.09.013

93. Takahashi T, Yamanaka T, Seto T, Harada H, Nokihara H, Saka H, et al. Prophylactic Cranial Irradiation Versus Observation in Patients With Extensive-Disease Small-Cell Lung Cancer: A Multicentre, Randomised, Open-Label, Phase 3 Trial. Lancet Oncol (2017) 18(5):663-71. doi: 10.1016/S1470-2045(17)30230-9

94. Yin X, Yan D, Qiu M, Huang L, Yan SX. Prophylactic Cranial Irradiation in Small Cell Lung Cancer: A Systematic Review and Meta-Analysis. BMC Cancer (2019) 19(1):95. doi: 10.1186/s12885-018-5251-3

95. Rusthoven CG, Yamamoto M, Bernhardt D, Smith DE, Gao D, Serizawa T, et al. Evaluation of First-Line Radiosurgery vs Whole-Brain Radiotherapy for Small Cell Lung Cancer Brain Metastases: The FIRE-SCLC Cohort Study. JAMA Oncol (2020) 6(7):1028-37. doi: 10.1001/jamaoncol. 2020.1271

96. Rodriguez de Dios N, Counago F, Murcia-Mejia M, Rico-Oses M, CalvoCrespo P, Samper P, et al. Randomized Phase III Trial of Prophylactic Cranial Irradiation With or Without Hippocampal Avoidance for Small-Cell Lung Cancer (PREMER): A GICOR-GOECP-SEOR Study. J Clin Oncol (2021) 39(28):3118-27. doi: 10.1200/JCO.21.00639

97. Bendell JC, Domchek SM, Burstein HJ, Harris L, Younger J, Kuter I, et al. Central Nervous System Metastases in Women Who Receive Trastuzumab- 
Based Therapy for Metastatic Breast Carcinoma. Cancer (2003) 97 (12):2972-7. doi: $10.1002 / \mathrm{cncr} .11436$

98. Lin NU, Winer EP. Brain Metastases: The HER2 Paradigm. Clin Cancer Res (2007) 13(6):1648-55. doi: 10.1158/1078-0432.CCR-06-2478

99. Kennecke H, Yerushalmi R, Woods R, Cheang MC, Voduc D, Speers CH, et al. Metastatic Behavior of Breast Cancer Subtypes. J Clin Oncol (2010) 28 (20):3271-7. doi: 10.1200/JCO.2009.25.9820

100. Slamon DJ, Leyland-Jones B, Shak S, Fuchs H, Paton V, Bajamonde A, et al. Use of Chemotherapy Plus a Monoclonal Antibody Against HER2 for Metastatic Breast Cancer That Overexpresses Her2. N Engl J Med (2001) 344(11):783-92. doi: 10.1056/NEJM200103153441101

101. Park YH, Park MJ, Ji SH, Yi SY, Lim DH, Nam DH, et al. Trastuzumab Treatment Improves Brain Metastasis Outcomes Through Control and Durable Prolongation of Systemic Extracranial Disease in HER2Overexpressing Breast Cancer Patients. Br J Cancer (2009) 100(6):894-900. doi: 10.1038/sj.bjc.6604941

102. Park IH, Ro J, Lee KS, Nam BH, Kwon Y, Shin KH. Trastuzumab Treatment Beyond Brain Progression in HER2-Positive Metastatic Breast Cancer. Ann Oncol (2009) 20(1):56-62. doi: 10.1093/annonc/mdn539

103. Okita Y, Narita Y, Suzuki T, Arita H, Yonemori K, Kinoshita T, et al. Extended Trastuzumab Therapy Improves the Survival of HER2-Positive Breast Cancer Patients Following Surgery and Radiotherapy for Brain Metastases. Mol Clin Oncol (2013) 1(6):995-1001. doi: 10.3892/mco.2013.162

104. Dawood S, Broglio K, Esteva FJ, Ibrahim NK, Kau SW, Islam R, et al. Defining Prognosis for Women With Breast Cancer and CNS Metastases by HER2 Status. Ann Oncol (2008) 19(7):1242-8. doi: 10.1093/annonc/ mdn036

105. Lin NU, Carey LA, Liu MC, Younger J, Come SE, Ewend M, et al. Phase II Trial of Lapatinib for Brain Metastases in Patients With Human Epidermal Growth Factor Receptor 2-Positive Breast Cancer. J Clin Oncol (2008) 26 (12):1993-9. doi: 10.1200/JCO.2007.12.3588

106. Lin NU, Diéras V, Paul D, Lossignol D, Christodoulou C, Stemmler HJ, et al. Multicenter Phase II Study of Lapatinib in Patients With Brain Metastases From HER2-Positive Breast Cancer. Clin Cancer Res (2009) 15(4):1452-9. doi: 10.1158/1078-0432.CCR-08-1080

107. Metro G, Foglietta J, Russillo M, Stocchi L, Vidiri A, Giannarelli D, et al. Clinical Outcome of Patients With Brain Metastases From HER2-Positive Breast Cancer Treated With Lapatinib and Capecitabine. Ann Oncol (2011) 22(3):625-30. doi: 10.1093/annonc/mdq434

108. Bachelot T, Romieu G, Campone M, Diéras V, Cropet C, Dalenc F, et al. Lapatinib Plus Capecitabine in Patients With Previously Untreated Brain Metastases From HER2-Positive Metastatic Breast Cancer (LANDSCAPE): A Single-Group Phase 2 Study. Lancet Oncol (2013) 14(1):64-71. doi: 10.1016/S1470-2045(12)70432-1

109. Freedman RA, Gelman RS, Anders CK, Melisko ME, Parsons HA, Cropp AM, et al. TBCRC 022: A Phase II Trial of Neratinib and Capecitabine for Patients With Human Epidermal Growth Factor Receptor 2-Positive Breast Cancer and Brain Metastases. J Clin Oncol (2019) 37(13):1081-9. doi: 10.1200/JCO.18.01511

110. Hurvitz SA, Saura C, Oliveira M, Trudeau ME, Moy B, Delaloge S, et al. Efficacy of Neratinib Plus Capecitabine in the Subgroup of Patients With Central Nervous System Involvement From the NALA Trial. Oncologist (2021) 26(18):e1327-38 doi: 10.1002/onco.13830

111. Lin NU, Borges V, Anders C, Murthy RK, Paplomata E, Hamilton E, et al. Intracranial Efficacy and Survival With Tucatinib Plus Trastuzumab and Capecitabine for Previously Treated HER2-Positive Breast Cancer With Brain Metastases in the HER2CLIMB Trial. J Clin Oncol (2020) 38 (23):2610-9. doi: 10.1200/JCO.20.00775

112. Bartsch R, Berghoff AS, Vogl U, Rudas M, Bergen E, Dubsky P, et al. Activity of T-DM1 in Her2-Positive Breast Cancer Brain Metastases. Clin Exp Metastasis (2015) 32(7):729-37. doi: 10.1007/s10585-015-9740-3

113. Jacot W, Pons E, Frenel JS, Guiu S, Levy C, Heudel PE, et al. Efficacy and Safety of Trastuzumab Emtansine (T-DM1) in Patients With HER2-Positive Breast Cancer With Brain Metastases. Breast Cancer Res Treat (2016) 157 (2):307-18. doi: 10.1007/s10549-016-3828-6

114. Krop IE, Lin NU, Blackwell K, Guardino E, Huober J, Lu M, et al. Trastuzumab Emtansine (T-DM1) Versus Lapatinib Plus Capecitabine in Patients With HER2-Positive Metastatic Breast Cancer and Central Nervous
System Metastases: A Retrospective, Exploratory Analysis in EMILIA. Ann Oncol (2015) 26(1):113-9. doi: 10.1093/annonc/mdu486

115. Montemurro F, Delaloge S, Barrios CH, Wuerstlein R, Anton A, Brain E, et al. Trastuzumab Emtansine (T-DM1) in Patients With HER2-Positive Metastatic Breast Cancer and Brain Metastases: Exploratory Final Analysis of Cohort 1 From KAMILLA, a Single-Arm Phase IIIb Clinical Trial(). Ann Oncol (2020) 31(10):1350-8. doi: 10.1016/j.annonc.2020.06.020

116. Bartsch R, Berghoff AS, Furtner J, Bergen ES, Roider-Schur S, Marhold M, et al. 280p Intracranial Activity of Trastuzumab-Deruxtecan (T-DXd) in HER2-Positive Breast Cancer Patients With Active Brain Metastases: Results From the First Stage of the Phase II TUXEDO-1 Trial. Ann Oncol (2021) 32: S486. doi: 10.1016/j.annonc.2021.08.563

117. Jerusalem GHM, Park YH, Yamashita T, Hurvitz SA, Modi S, Andre F, et al. Trastuzumab Deruxtecan (T-DXd) in Patients With HER2+ Metastatic Breast Cancer With Brain Metastases: A Subgroup Analysis of the DESTINY-Breast01 Trial. J Clin Oncol (2021) 39(15_suppl):526-. doi: 10.1200/JCO.2021.39.15_suppl.526

118. Tolaney SM, Sahebjam S, Le Rhun E, Bachelot T, Kabos P, Awada A, et al. A Phase II Study of Abemaciclib in Patients With Brain Metastases Secondary to Hormone Receptor-Positive Breast Cancer. Clin Cancer Res (2020) 26 (20):5310. doi: 10.1158/1078-0432.CCR-20-1764

119. Brastianos PK, Kim AE, Wang N, Lee EQ, Ligibel J, Cohen JV, et al. Palbociclib Demonstrates Intracranial Activity in Progressive Brain Metastases Harboring Cyclin-Dependent Kinase Pathway Alterations. Nat Cancer (2021) 2(5):498-502. doi: 10.1038/s43018-021-00198-5

120. Anders C, Deal AM, Abramson V, Liu MC, Storniolo AM, Carpenter JT, et al. TBCRC 018: Phase II Study of Iniparib in Combination With Irinotecan to Treat Progressive Triple Negative Breast Cancer Brain Metastases. Breast Cancer Res Treat (2014) 146(3):557-66. doi: 10.1007/ s10549-014-3039-y

121. Litton JK, Hurvitz SA, Mina LA, Rugo HS, Lee KH, Gonçalves A, et al. Talazoparib Versus Chemotherapy in Patients With Germline BRCA1/2Mutated HER2-Negative Advanced Breast Cancer: Final Overall Survival Results From the EMBRACA Trial. Ann Oncol (2020) 31(11):1526-35. doi: 10.1016/j.annonc.2020.08.2098

122. Solomon BJ, Cappuzzo F, Felip E, Blackhall FH, Costa DB, Kim DW, et al. Intracranial Efficacy of Crizotinib Versus Chemotherapy in Patients With Advanced ALK-Positive Non-Small-Cell Lung Cancer: Results From PROFILE 1014. J Clin Oncol (2016) 34(24):2858-65. doi: 10.1200/ JCO.2015.63.5888

123. Crinò L, Ahn M-J, De Marinis F, Groen HJM, Wakelee H, Hida T, et al. Multicenter Phase II Study of Whole-Body and Intracranial Activity With Ceritinib in Patients With ALK-Rearranged Non-Small-Cell Lung Cancer Previously Treated With Chemotherapy and Crizotinib: Results From ASCEND-2. J Clin Oncol (2016) 34(24):2866-73. doi: 10.1200/JCO.2015.65.5936

124. Gadgeel SM, Gandhi L, Riely GJ, Chiappori AA, West HL, Azada MC, et al. Safety and Activity of Alectinib Against Systemic Disease and Brain Metastases in Patients With Crizotinib-Resistant ALK-Rearranged NonSmall-Cell Lung Cancer (AF-002JG): Results From the Dose-Finding Portion of a Phase 1/2 Study. Lancet Oncol (2014) 15(10):1119-28. doi: 10.1016/S1470-2045(14)70362-6

125. Peters S, Camidge DR, Shaw AT, Gadgeel S, Ahn JS, Kim DW, et al. Alectinib Versus Crizotinib in Untreated ALK-Positive Non-Small-Cell Lung Cancer. N Engl J Med (2017) 377(9):829-38. doi: 10.1056/NEJMoa1704795

126. Camidge DR, Kim HR, Ahn MJ, Yang JC, Han JY, Lee JS, et al. Brigatinib Versus Crizotinib in ALK-Positive Non-Small-Cell Lung Cancer. N Engl J Med (2018) 379(21):2027-39. doi: 10.1056/NEJMoa1810171

127. Shaw AT, Bauer TM, De Marinis F, Felip E, Goto Y, Liu G, et al. First-Line Lorlatinib or Crizotinib in Advanced ALK-Positive Lung Cancer. N Engl J Med (2020) 383(21):2018-29. doi: 10.1056/NEJMoa2027187

128. Shaw AT, Solomon BJ, Chiari R, Riely GJ, Besse B, Soo RA, et al. Lorlatinib in Advanced ROS1-Positive Non-Small-Cell Lung Cancer: A Multicentre, Open-Label, Single-Arm, Phase 1-2 Trial. Lancet Oncol (2019) 20 (12):1691-701. doi: 10.1016/S1470-2045(19)30655-2

129. Horn L, Wang Z, Wu G, Poddubskaya E, Mok T, Reck M, et al. Ensartinib vs Crizotinib for Patients With Anaplastic Lymphoma Kinase-Positive NonSmall Cell Lung Cancer: A Randomized Clinical Trial. JAMA Oncol (2021) 7 (11):1617-25. doi: 10.1001/jamaoncol.2021.3523 
130. Ahn MJ, Han JY, Lee KH, Kim SW, Kim DW, Lee YG, et al. Lazertinib in Patients With EGFR Mutation-Positive Advanced Non-Small-Cell Lung Cancer: Results From the Dose Escalation and Dose Expansion Parts of a First-in-Human, Open-Label, Multicentre, Phase 1-2 Study. Lancet Oncol (2019) 20(12):1681-90. doi: 10.1016/S1470-2045(19)30504-2

131. Cho BC, Han JY, Kim SW, Lee KH, Cho EK, Lee YG, et al. A Phase 1/2 Study of Lazertinib $240 \mathrm{Mg}$ in Patients With Advanced EGFR T790M-Positive NSCLC After Previous EGFR Tyrosine Kinase Inhibitors. J Thorac Oncol (2021). doi: 10.1016/j.jtho.2021.11.025

132. Shi Y, Zhang S, Hu X, Feng J, Ma Z, Zhou J, et al. Safety, Clinical Activity, and Pharmacokinetics of Alflutinib (AST2818) in Patients With Advanced NSCLC With EGFR T790M Mutation. J Thorac Oncol (2020) 15(6):1015-26. doi: 10.1016/j.jtho.2020.01.010

133. Shi Y, Hu X, Zhang S, Lv D, Wu L, Yu Q, et al. Efficacy, Safety, and Genetic Analysis of Furmonertinib (AST2818) in Patients With EGFR T790M Mutated Non-Small-Cell Lung Cancer: A Phase 2b, Multicentre, SingleArm, Open-Label Study. Lancet Respir Med (2021) 9(8):829-39. doi: 10.1016/S2213-2600(20)30455-0

134. Park K, Haura EB, Leighl NB, Mitchell P, Shu CA, Girard N, et al. Amivantamab in EGFR Exon 20 Insertion-Mutated Non-Small-Cell Lung Cancer Progressing on Platinum Chemotherapy: Initial Results From the CHRYSALIS Phase I Study. J Clin Oncol (2021) 39(30):3391-402. doi: $10.1200 /$ JCO.21.00662

135. Ceresoli GL, Cappuzzo F, Gregorc V, Bartolini S, Crinò L, Villa E. Gefitinib in Patients With Brain Metastases From Non-Small-Cell Lung Cancer: A Prospective Trial. Ann Oncol (2004) 15(7):1042-7. doi: 10.1093/annonc/mdh276

136. Hotta K, Kiura K, Ueoka H, Tabata M, Fujiwara K, Kozuki T, et al. Effect of Gefitinib ('Iressa', ZD1839) on Brain Metastases in Patients With Advanced Non-Small-Cell Lung Cancer. Lung Cancer (2004) 46(2):255-61. doi: 10.1016/j.lungcan.2004.04.036

137. Lee DH, Han J-Y, Lee HG, Lee JJ, Lee EK, Kim HY, et al. Gefitinib as a FirstLine Therapy of Advanced or Metastatic Adenocarcinoma of the Lung in Never-Smokers. Clin Cancer Res (2005) 11(8):3032-7. doi: 10.1158/10780432.CCR-04-2149

138. Chiu C-H, Tsai C-M, Chen Y-M, Chiang S-C, Liou J-L, Perng R-P. Gefitinib is Active in Patients With Brain Metastases From Non-Small Cell Lung Cancer and Response is Related to Skin Toxicity. Lung Cancer (2005) 47 (1):129-38. doi: 10.1016/j.lungcan.2004.05.014

139. Kim J-E, Lee DH, Choi Y, Yoon DH, Kim S-W, Suh C, et al. Epidermal Growth Factor Receptor Tyrosine Kinase Inhibitors as a First-Line Therapy for Never-Smokers With Adenocarcinoma of the Lung Having Asymptomatic Synchronous Brain Metastasis. Lung Cancer (2009) 65 (3):351-4. doi: 10.1016/j.lungcan.2008.12.011

140. Park SJ, Kim HT, Lee DH, Kim KP, Kim SW, Suh C, et al. Efficacy of Epidermal Growth Factor Receptor Tyrosine Kinase Inhibitors for Brain Metastasis in Non-Small Cell Lung Cancer Patients Harboring Either Exon 19 or 21 Mutation. Lung Cancer (2012) 77(3):556-60. doi: 10.1016/ j.lungcan.2012.05.092

141. Mok TS, Wu YL, Ahn MJ, Garassino MC, Kim HR, Ramalingam SS, et al. Osimertinib or Platinum-Pemetrexed in EGFR T790M-Positive Lung Cancer. N Engl J Med (2017) 376(7):629-40. doi: 10.1056/ NEJMoa1612674

142. Soria JC, Ohe Y, Vansteenkiste J, Reungwetwattana T, Chewaskulyong B, Lee $\mathrm{KH}$, et al. Osimertinib in Untreated EGFR-Mutated Advanced Non-SmallCell Lung Cancer. N Engl J Med (2018) 378(2):113-25. doi: 10.1056/ NEJMoa1713137

143. Skoulidis F, Li BT, Dy GK, Price TJ, Falchook GS, Wolf J, et al. Sotorasib for Lung Cancers With KRAS P.G12C Mutation. N Engl J Med (2021) 384 (25):2371-81. doi: 10.1056/NEJMoa2103695

144. Drilon A, Oxnard GR, Tan DSW, Loong HHF, Johnson M, Gainor J, et al. Efficacy of Selpercatinib in RET Fusion-Positive Non-Small-Cell Lung Cancer. N Engl J Med (2020) 383(9):813-24. doi: 10.1056/NEJMoa2005653

145. Gainor JF, Curigliano G, Kim DW, Lee DH, Besse B, Baik CS, et al. Pralsetinib for RET Fusion-Positive Non-Small-Cell Lung Cancer (ARROW): A Multi-Cohort, Open-Label, Phase 1/2 Study. Lancet Oncol (2021) 22(7):959-69. doi: 10.1016/S1470-2045(21)00247-3

146. Drilon A, Ou SI, Cho BC, Kim DW, Lee J, Lin JJ, et al. Repotrectinib (TPX0005) Is a Next-Generation ROS1/TRK/ALK Inhibitor That Potently
Inhibits ROS1/TRK/ALK Solvent- Front Mutations. Cancer Discov (2018) 8(10):1227-36. doi: 10.1158/2159-8290.CD-18-0484

147. Long GV, Trefzer U, Davies MA, Kefford RF, Ascierto PA, Chapman PB, et al. Dabrafenib in Patients With Val600Glu or Val600Lys BRAF-Mutant Melanoma Metastatic to the Brain (BREAK-MB): A Multicentre, OpenLabel, Phase 2 Trial. Lancet Oncol (2012) 13(11):1087-95. doi: 10.1016/ S1470-2045(12)70431-X

148. Davies MA, Saiag P, Robert C, Grob JJ, Flaherty KT, Arance A, et al. Dabrafenib Plus Trametinib in Patients With BRAF(V600)-Mutant Melanoma Brain Metastases (COMBI-MB): A Multicentre, Multicohort, Open-Label, Phase 2 Trial. Lancet Oncol (2017) 18(7):863-73. doi: 10.1016/S1470-2045(17)30429-1

149. McArthur GA, Maio M, Arance A, Nathan P, Blank C, Avril MF, et al. Vemurafenib in Metastatic Melanoma Patients With Brain Metastases: An Open-Label, Single-Arm, Phase 2, Multicentre Study. Ann Oncol (2017) 28 (3):634-41. doi: 10.1093/annonc/mdw641

150. Arvanitis CD, Ferraro GB, Jain RK. The Blood-Brain Barrier and BloodTumour Barrier in Brain Tumours and Metastases. Nat Rev Cancer (2020) 20 (1):26-41. doi: 10.1038/s41568-019-0205-x

151. Musolino A, Ciccolallo L, Panebianco M, Fontana E, Zanoni D, Bozzetti C, et al. Multifactorial Central Nervous System Recurrence Susceptibility in Patients With HER2-Positive Breast Cancer. Cancer (2011) 117(9):1837-46. doi: $10.1002 /$ cncr. 25771

152. Pestalozzi BC, Brignoli S. Trastuzumab in CSF. J Clin Oncol (2000) 18 (11):2349-51. doi: 10.1200/JCO.2000.18.11.2349

153. Nguyen LV, Searle K, Jerzak KJ. Central Nervous System-Specific Efficacy of CDK4/6 Inhibitors in Randomized Controlled Trials for Metastatic Breast Cancer. Oncotarget (2019) 10(59):6317-22. doi: 10.18632/oncotarget. 27238

154. Schlam I, Tolaney SM. Is There a Role for CDK $4 / 6$ Inhibitors in Breast Cancer Brain Metastases? Oncotarget (2021) 12(9):873-5. doi: 10.18632/ oncotarget.27904

155. Lin NU, Claus E, Sohl J, Razzak AR, Arnaout A, Winer EP. Sites of Distant Recurrence and Clinical Outcomes in Patients With Metastatic TripleNegative Breast Cancer: High Incidence of Central Nervous System Metastases. Cancer (2008) 113(10):2638-45. doi: 10.1002/cncr.23930

156. Guérin A, Sasane M, Zhang J, Culver KW, Dea K, Nitulescu R, et al. Brain Metastases in Patients With ALK+ Non-Small Cell Lung Cancer: Clinical Symptoms, Treatment Patterns and Economic Burden. J Med Economics (2015) 18(4):312-22. doi: 10.3111/13696998.2014.1003644

157. Kelly WJ, Shah NJ, Subramaniam DS. Management of Brain Metastases in Epidermal Growth Factor Receptor Mutant Non-Small-Cell Lung Cancer. Front Oncol (2018) 8:208. doi: 10.3389/fonc.2018.00208

158. Jänne PA, Yang JC, Kim DW, Planchard D, Ohe Y, Ramalingam SS, et al. AZD9291 in EGFR Inhibitor-Resistant Non-Small-Cell Lung Cancer. N Engl J Med (2015) 372(18):1689-99. doi: 10.1056/NEJMoa1411817

159. Melosky B, Banerji S, Blais N, Chu Q, Juergens R, Leighl NB, et al. Canadian Consensus: A New Systemic Treatment Algorithm for Advanced EGFRMutated Non-Small-Cell Lung Cancer. Curr Oncol (2020) 27(2):e146-e55. doi: $10.3747 /$ co.27.6007

160. Tomasini P, Walia P, Labbe C, Jao K, Leighl NB. Targeting the KRAS Pathway in Non-Small Cell Lung Cancer. Oncologist (2016) 21(12):1450-60. doi: 10.1634/theoncologist.2015-0084

161. Paik PK, Felip E, Veillon R, Sakai H, Cortot AB, Garassino MC, et al. Tepotinib in Non-Small-Cell Lung Cancer With MET Exon 14 Skipping Mutations. N Engl J Med (2020) 383(10):931-43. doi: 10.1056/ NEJMoa2004407

162. Wolf J, Seto T, Han JY, Reguart N, Garon EB, Groen HJM, et al. Capmatinib in MET Exon 14-Mutated or MET-Amplified Non-Small-Cell Lung Cancer. N Engl J Med (2020) 383(10):944-57. doi: 10.1056/NEJMoa2002787

163. Hong DS, DuBois SG, Kummar S, Farago AF, Albert CM, Rohrberg KS, et al. Larotrectinib in Patients With TRK Fusion-Positive Solid Tumours: A Pooled Analysis of Three Phase 1/2 Clinical Trials. Lancet Oncol (2020) 21 (4):531-40. doi: 10.1016/S1470-2045(19)30856-3

164. John T, Chiu CH, Cho BC, Fakih M, Farago AF, Demetri GD, et al. 364o Intracranial Efficacy of Entrectinib in Patients With NTRK Fusion-Positive Solid Tumours and Baseline CNS Metastases. Ann Oncol (2020) 31:S397-S8. doi: 10.1016/j.annonc.2020.08.473 
165. Davies MA, Liu P, McIntyre S, Kim KB, Papadopoulos N, Hwu W-J, et al. Prognostic Factors for Survival in Melanoma Patients With Brain Metastases. Cancer (2011) 117(8):1687-96. doi: 10.1002/cncr.25634

166. Jakob JA, Bassett RL, Ng CS, Curry JL, Joseph RW, Alvarado GC, et al. NRAS Mutation Status Is an Independent Prognostic Factor in Metastatic Melanoma. Cancer (2012) 118(16):4014-23. doi: 10.1002/cncr.26724

167. Wilson EH, Weninger W, Hunter CA. Trafficking of Immune Cells in the Central Nervous System. J Clin Invest (2010) 120(5):1368-79. doi: 10.1172/ JCI41911

168. Prins RM, Vo DD, Khan-Farooqi H, Yang MY, Soto H, Economou JS, et al. NK and CD4 Cells Collaborate to Protect Against Melanoma Tumor Formation in the Brain. J Immunol (2006) 177(12):8448-55. doi: 10.4049/ jimmunol.177.12.8448

169. Margolin K, Ernstoff MS, Hamid O, Lawrence D, McDermott D, Puzanov I, et al. Ipilimumab in Patients With Melanoma and Brain Metastases: An Open-Label, Phase 2 Trial. Lancet Oncol (2012) 13(5):459-65. doi: 10.1016/ S1470-2045(12)70090-6

170. Goldberg SB, Gettinger SN, Mahajan A, Chiang AC, Herbst RS, Sznol M, et al. Pembrolizumab for Patients With Melanoma or Non-Small-Cell Lung Cancer and Untreated Brain Metastases: Early Analysis of a NonRandomised, Open-Label, Phase 2 Trial. Lancet Oncol (2016) 17(7):97683. doi: 10.1016/S1470-2045(16)30053-5

171. Crinò L, Bronte G, Bidoli P, Cravero P, Minenza E, Cortesi E, et al. Nivolumab and Brain Metastases in Patients With Advanced NonSquamous Non-Small Cell Lung Cancer. Lung Cancer (2019) 129:35-40. doi: 10.1016/j.lungcan.2018.12.025

172. Tawbi HA, Forsyth PA, Algazi A, Hamid O, Hodi FS, Moschos SJ, et al. Combined Nivolumab and Ipilimumab in Melanoma Metastatic to the Brain. N Engl J Med (2018) 379(8):722-30. doi: 10.1056/ NEJMoa 1805453

173. Tawbi HA, Forsyth PA, Hodi FS, Algazi AP, Hamid O, Lao CD, et al. LongTerm Outcomes of Patients With Active Melanoma Brain Metastases Treated With Combination Nivolumab Plus Ipilimumab (CheckMate 204): Final Results of an Open-Label, Multicentre, Phase 2 Study. Lancet Oncol (2021) 22(12):1692-704. doi: 10.1016/S1470-2045(21)00545-3

174. Tawbi HA, Schadendorf D, Lipson EJ, Ascierto PA, Matamala L, Castillo Gutiérrez E, et al. Relatlimab and Nivolumab Versus Nivolumab in Untreated Advanced Melanoma. N Engl J Med (2022) 386(1):24-34. doi: 10.1056/NEJMoa2109970

175. Schmid P, Adams S, Rugo HS, Schneeweiss A, Barrios CH, Iwata H, et al. Atezolizumab and Nab-Paclitaxel in Advanced Triple-Negative Breast Cancer. N Engl J Med (2018) 379(22):2108-21. doi: 10.1056/NEJMoa1809615

176. Schmid P, Rugo HS, Adams S, Schneeweiss A, Barrios CH, Iwata H, et al. Atezolizumab Plus Nab-Paclitaxel as First-Line Treatment for Unresectable, Locally Advanced or Metastatic Triple-Negative Breast Cancer (IMpassion130): Updated Efficacy Results From a Randomised, DoubleBlind, Placebo-Controlled, Phase 3 Trial. Lancet Oncol (2020) 21(1):44-59. doi: 10.1016/S1470-2045(19)30689-8

177. Flippot R, Dalban C, Laguerre B, Borchiellini D, Gravis G, Négrier S, et al. Safety and Efficacy of Nivolumab in Brain Metastases From Renal Cell Carcinoma: Results of the GETUG-AFU 26 NIVOREN Multicenter Phase II Study. J Clin Oncol (2019) 37(23):2008-16. doi: 10.1200/JCO.18.02218

178. Knisely JP, Yu JB, Flanigan J, Sznol M, Kluger HM, Chiang VL. Radiosurgery for Melanoma Brain Metastases in the Ipilimumab Era and the Possibility of Longer Survival. J Neurosurg (2012) 117(2):227-33. doi: 10.3171/ 2012.5.JNS111929

179. Silk AW, Bassetti MF, West BT, Tsien CI, Lao CD. Ipilimumab and Radiation Therapy for Melanoma Brain Metastases. Cancer Med (2013) 2 (6):899-906. doi: 10.1002/cam4.140

180. Mathew M, Tam M, Ott PA, Pavlick AC, Rush SC, Donahue BR, et al. Ipilimumab in Melanoma With Limited Brain Metastases Treated With Stereotactic Radiosurgery. Melanoma Res (2013) 23(3):191-5. doi: 10.1097/ CMR.0b013e 32835f3d90

181. Minniti G, Anzellini D, Reverberi C, Cappellini GCA, Marchetti L, Bianciardi F, et al. Stereotactic Radiosurgery Combined With Nivolumab or Ipilimumab for Patients With Melanoma Brain Metastases: Evaluation of Brain Control and Toxicity. J ImmunoTher Cancer (2019) 7(1):102. doi: 10.1186/s40425-019-0588-y
182. Okwan-Duodu D, Pollack BP, Lawson D, Khan MK. Role of Radiation Therapy as Immune Activator in the Era of Modern Immunotherapy for Metastatic Malignant Melanoma. Am J Clin Oncol (2015) 38(1):119-25. doi: 10.1097/COC.0b013e3182940dc3

183. Seung SK, Curti BD, Crittenden M, Walker E, Coffey T, Siebert JC, et al. Phase 1 Study of Stereotactic Body Radiotherapy and Interleukin-2-Tumor and Immunological Responses. Sci Transl Med (2012) 4(137):137ra74. doi: 10.1126/scitranslmed.3003649

184. Bot I, Blank CU, Brandsma D. Clinical and Radiological Response of Leptomeningeal Melanoma After Whole Brain Radiotherapy and Ipilimumab. J Neurol (2012) 259(9):1976-8. doi: 10.1007/s00415-012-6488-4

185. Mueller-Brenne T, Rudolph B, Schmidberger H, Grabbe S, Loquai C. Successful Therapy of a Cerebral Metastasized Malign Melanoma by Whole-Brain-Radiation and Therapy With Ipilimumab. In: Journal Der Deutschen Dermatologischen Gesellschaft. 350 MAIN ST, MALDEN 02148, MA USA: WILEY-BLACKWELL COMMERCE PLACE (2011).

186. Sperduto PW, Wang M, Robins HI, Schell MC, Werner-Wasik M, Komaki R, et al. A Phase 3 Trial of Whole Brain Radiation Therapy and Stereotactic Radiosurgery Alone Versus WBRT and SRS With Temozolomide or Erlotinib for Non-Small Cell Lung Cancer and 1 to 3 Brain Metastases: Radiation Therapy Oncology Group 0320. Int J Radiat Oncol Biol Phys (2013) 85(5):1312-8. doi: 10.1016/j.ijrobp.2012.11.042

187. Brastianos PK, Carter SL, Santagata S, Cahill DP, Taylor-Weiner A, Jones RT, et al. Genomic Characterization of Brain Metastases Reveals Branched Evolution and Potential Therapeutic Targets. Cancer Discov (2015) 5 (11):1164-77. doi: 10.1158/2159-8290.CD-15-0369

188. Luo YH, Luo L, Wampfler JA, Wang Y, Liu D, Chen YM, et al. 5-Year Overall Survival in Patients With Lung Cancer Eligible or Ineligible for Screening According to US Preventive Services Task Force Criteria: A Prospective, Observational Cohort Study. Lancet Oncol (2019) 20(8):1098-108. doi: 10.1016/S1470-2045(19)30329-8

189. Schunemann HJ, Lerda D, Quinn C, Follmann M, Alonso-Coello P, Rossi PG, et al. Breast Cancer Screening and Diagnosis: A Synopsis of the European Breast Guidelines. Ann Intern Med (2020) 172(1):46-56. doi: 10.7326/M19-2125

190. Carroll PH, Mohler JL. NCCN Guidelines Updates: Prostate Cancer and Prostate Cancer Early Detection. J Natl Compr Canc Netw (2018) 16 (5S):620-3. doi: 10.6004/jnccn.2018.0036

191. Vernooij MW, Ikram MA, Tanghe HL, Vincent AJ, Hofman A, Krestin GP, et al. Incidental Findings on Brain MRI in the General Population. $N$ Engl $J$ Med (2007) 357(18):1821-8. doi: 10.1056/NEJMoa070972

192. O'Sullivan JW, Muntinga T, Grigg S, Ioannidis JPA. Prevalence and Outcomes of Incidental Imaging Findings: Umbrella Review. BMJ (2018) 361:k2387. doi: 10.1136/bmj.k2387

193. Komorowski AS, Warner E, MacKay HJ, Sahgal A, Pritchard KI, Jerzak KJ. Incidence of Brain Metastases in Nonmetastatic and Metastatic Breast Cancer: Is There a Role for Screening? Clin Breast Cancer (2020) 20(1): e54-64. doi: 10.1016/j.clbc.2019.06.007

194. Le Rhun E, Guckenberger M, Smits M, Dummer R, Bachelot T, Sahm F, et al. EANO-ESMO Clinical Practice Guidelines for Diagnosis, Treatment and Follow-Up of Patients With Brain Metastasis From Solid Tumours. Ann Oncol (2021) 32(11):1332-47. doi: 10.1016/j.annonc.2021.07.016

195. Kuksis M, Gao Y, Tran W, Hoey C, Kiss A, Komorowski AS, et al. The Incidence of Brain Metastases Among Patients With Metastatic Breast Cancer: A Systematic Review and Meta-Analysis. Neuro Oncol (2021) 23 (6):894-904. doi: 10.1093/neuonc/noaa285

196. Boire A, Brandsma D, Brastianos PK, Le Rhun E, Ahluwalia M, Junck L, et al. Liquid Biopsy in Central Nervous System Metastases: A RANO Review and Proposals for Clinical Applications. Neuro Oncol (2019) 21(5):571-84. doi: 10.1093/neuonc/noz012

197. Seoane J, De Mattos-Arruda L, Le Rhun E, Bardelli A, Weller M. Cerebrospinal Fluid Cell-Free Tumour DNA as a Liquid Biopsy for Primary Brain Tumours and Central Nervous System Metastases. Ann Oncol (2019) 30(2):211-8. doi: 10.1093/annonc/mdy544

198. Shih DJH, Nayyar N, Bihun I, Dagogo-Jack I, Gill CM, Aquilanti E, et al. Genomic Characterization of Human Brain Metastases Identifies Drivers of Metastatic Lung Adenocarcinoma. Nat Genet (2020) 52(4):371-7. doi: 10.1038/s41588-020-0592-7 
199. Iwamoto T, Niikura N, Ogiya R, Yasojima H, Watanabe KI, Kanbayashi C, et al. Distinct Gene Expression Profiles Between Primary Breast Cancers and Brain Metastases From Pair-Matched Samples. Sci Rep (2019) 9(1):13343. doi: 10.1038/s41598-019-50099-y

200. Fischer GM, Jalali A, Kircher DA, Lee WC, McQuade JL, Haydu LE, et al. Molecular Profiling Reveals Unique Immune and Metabolic Features of Melanoma Brain Metastases. Cancer Discov (2019) 9(5):628-45. doi: 10.1158/2159-8290.CD-18-1489

201. Hanssen A, Riebensahm C, Mohme M, Joosse SA, Velthaus JL, Berger LA, et al. Frequency of Circulating Tumor Cells (CTC) in Patients With Brain Metastases: Implications as a Risk Assessment Marker in Oligo-Metastatic Disease. Cancers (Basel) (2018) 10(12):527. doi: 10.3390/cancers10120527

202. Zeng Q, Michael IP, Zhang P, Saghafinia S, Knott G, Jiao W, et al. Synaptic Proximity Enables NMDAR Signalling to Promote Brain Metastasis. Nature (2019) 573(7775):526-31. doi: 10.1038/s41586-019-1576-6

203. Gril B, Paranjape AN, Woditschka S, Hua E, Dolan EL, Hanson J, et al. Reactive Astrocytic S1P3 Signaling Modulates the Blood-Tumor Barrier in Brain Metastases. Nat Commun (2018) 9(1):2705. doi: 10.1038/s41467-01805030-w

Conflict of Interest: SD acts as a consultant for Medexus and is on the advisory board for the Subcortical Surgery Group and Xpan Medical as well as the Speaker's
Board for the Congress of Neurological Surgeons, American Association of Neurological Surgeons, and Society for NeuroOncology. KJ is a speaker/advisor board/consultant for: Amgen, Astra Zeneca, Apo Biologix, Eli Lilly, Esai, Exact Sciences, Knight Therapeutics, Pfizer, Merck, Novartis, Purdue Pharma, Roche, Seagen, Viatris; and receives research funding from Eli Lilly, Astra Zeneca.

The remaining authors declare that the research was conducted in the absence of any commercial or financial relationships that could be construed as a potential conflict of interest.

Publisher's Note: All claims expressed in this article are solely those of the authors and do not necessarily represent those of their affiliated organizations, or those of the publisher, the editors and the reviewers. Any product that may be evaluated in this article, or claim that may be made by its manufacturer, is not guaranteed or endorsed by the publisher.

Copyright $\odot 2022$ Li, Gaebe, Jerzak, Cheema, Sahgal and Das. This is an open-access article distributed under the terms of the Creative Commons Attribution License (CC BY). The use, distribution or reproduction in other forums is permitted, provided the original author(s) and the copyright owner(s) are credited and that the original publication in this journal is cited, in accordance with accepted academic practice. No use, distribution or reproduction is permitted which does not comply with these terms. 